\title{
A finite element model based on coupled refined high-order global-local theory for static analysis of electromechanical embedded shear-mode piezoelectric sandwich composite beams with various widths
}

\author{
S.B. Beheshti-Aval ${ }^{\text {a,*}}$, S. Shahvaghar-Asl ${ }^{\text {b }}$, M. Lezgy-Nazargah ${ }^{c}$, M. Noori ${ }^{\mathrm{d}}$ \\ a Civil Engineering Faculty, K.N. Toosi University of Technology, Tehran, Iran \\ ${ }^{\mathrm{b}}$ Civil Engineering Faculty, K.N. Toosi University of Technology, Tehran, Iran \\ ${ }^{\mathrm{c}}$ Hakim Sabzevari University, Sabzevar, Iran \\ d College of Engineering, California Polytechnic State University, San Luis Obispo, California, USA
}

\begin{abstract}
A B S T R A C T
In this study, a new theory for the accurate simulation of the shear-mode behaviour of thin or thick piezoelectric sandwich composite beams is developed. The effects of transverse normal stress and transverse flexibility of layers are considered in the development of the proposed formulation. In order to increase the computational accuracy, all kinematic and stress continuity conditions are satisfied at layer interfaces. Moreover, for the first time, both the electrically induced strain components and the transverse flexibility are taken into account in the proposed formulation. Despite the fact that the number of unknown mechanical parameters in this theory is only one degree higher than the first order shear deformation theory, the accuracy is surprisingly more pronounced for the thicker beams.
\end{abstract}

\section{Introduction}

In recent years, piezoelectric materials have been widely used in the construction of smart structures. These electromechanical materials are able to control the responses of structures subjected to dynamic loading through sensing and actuating. A wide range of application for these materials is recognized in high-tech industries. Conventionally, piezoelectric actuators in a smart structure are inserted in the direction of the thickness and are usually bonded to the surfaces of the host structure. Such surface bonded actuators that induce longitudinal strains are known as extension-mode piezoelectric. Due to their inherent brittleness installing on external surface of host structures, where vulnerable to impact loads, a new adaptive sandwich structure has been suggested in which an axially poled piezoelectric layer was used between substrate layers. With the aid of the shear properties of piezoelectric materials and through applying external electric field in the thickness direction, this new approach makes it possible to induce shear strain in actuator layers and consequently gain the desired deformations.

An extensive body of research has been carried out describing the extension-mode piezoelectric actuators. Various mathematical models have been presented for structures containing extension-mode sensors and actuators. Crawley and de Luis [1], Tzou and Gadre [2], Wang and Rogers [3] and Sung et al. [4] used induced strain models for the representation of actuator responses of piezoelectric materials. By solving the governing differential equations for the three-dimensional (3D) theory of piezoelectricity, Brooks and Heyliger [5], and Ray et al. [6,7] obtained exact solutions for static analysis of extension-mode piezoelectric laminates in cylindrical bending. However, development of these solutions is a difficult task and the resulting solution cannot be expressed in a closed form for the general cases of arbitrary geometry, boundary and loading conditions. Moreover, the cost of the 3D finite element analysis is relatively high, and it poses a problem when piezoelectric layers are thin compared to the structure size. Hwang and Park [8], Suleman and Venkaya [9], Sheikh et al.

\footnotetext{
* Corresponding author. Tel.: +98 21 88779476; fax: +98 2188786215.

E-mail addresses: beheshti@kntu.ac.ir, sb_beheshti@yahoo.com (S.B. Beheshti-Aval).
} 
[10], Kogl and Bucalem [11,12] Chee et al. [13], Jiang and Li[14], Shu [15], Thornburgh and Chattopadhyay [16], Fukunaga et al. [17] and Mitchell and Reddy [18]used equivalent single layer theories (ESLT) for the analysis of extension-mode piezoelectric beams/plates structures. The idea of layer-wise theory (LWT) or discrete-layer theory was presented and developed by Heyliger and Saravanos [19], Saravanos and Heyliger [20], Saravanos et al.[21], Kusculuoglu et al. [22], Garcia Lage et al. [23,24] Robaldo et al. [25], and Tzou and Ye [26]. Beheshti-Aval and Lezgy-Nazargah[27,28] used a sinus zigzag (ZZ) model for analyzing and controlling of smart laminated piezoelectric beams. Kapuria [29], Kapuria et al. [30], and Kapuria and Alam[31] presented an efficient coupled ZZ theory for static and dynamic analysis of extension-mode piezoelectric laminated/sandwich beams based on third order $\mathrm{ZZ}$ approximations. Beheshti-Aval et al. [32] introduced a computationally low cost FE model for the static analysis of extension-mode piezoelectric multilayered/sandwich beams based on global-local theory. For an overview on the modeling of extension-mode piezoelectric laminates, readers can refer to Benjeddou[33].

The use of a shear-mode piezoelectric actuator was first proposed by Sun and Zhang [34]. In their work, they employed thickness-shear piezoelectric patches to create transverse deflection in sandwich beams. Moreover, they demonstrated that shear-mode actuators can offer many advantages over the extension-mode actuators. The analysis of this study was carried out using the ANSYS finite-element package. Furthermore, Zhang and Sun [35] developed an analytical model to predict the static, as well as the dynamic behavior of a sandwich beam which comprises of a core layer of shear-mode piezoelectric. The model relies on the assumptions that the face layers obey the EulerBernoulli theory, while the core piezoelectric layer follows the Timoshenko theory. Moreover, closed-form solutions of a static cantilever beam with shear-mode actuators were presented. Benjeddou et al. [36] proposed a finite element model of a sandwich beam with extension and shear piezoelectric segments. To verify their model, they compared their finite element results with those obtained by Zhang andSun. In addition, they presented the dynamic characteristics (i.e. natural bending frequencies and mode shapes) of two sandwich beams; one with a shear-mode actuator and another with extension-mode actuators. Raja et al. [37] extended the finite element model of Benjeddou's research team to include a vibration control scheme. Aldraihem and Khdeir [38-41] proposed analytical models and analytical solutions for beams with shear and extension piezoelectric actuators. The models proposed by these researchers are based on the first-order beam theory and higher-order beam theory. The analytical solutions are obtained using the state-space approach along with the Jordan canonical form. The deflections of the beams with various boundary conditions were investigated. Vel and Batra [42] obtained an exact 3D state-space solution for the static cylindrical bending of simply supported composite beams with shear-mode piezoelectric actuators.

Most of the studies on the modeling of laminated sandwich structures have focused on the extension-mode piezoelectric sensors and actuators. In comparison with the extension-mode piezoelectric, the modeling of structures with embedded shear-mode piezoelectric layers is still an unresolved challenge. The available theories for the laminated beams with shear-mode piezoelectric layers either do not consider the transverse flexibility or do not impose the continuity conditions of the transverse normal stresses at the interfaces. However, the transverse normal stresses and strains and the transverse flexibility have important roles in the analysis of such structures, especially those with soft cores. The continuity conditions of the transverse shear stresses are also neglected in most of the available models. Moreover, the available theories for the beams/plates with shear-mode piezoelectric layers neglect the in-plane electric field components. Due to direct piezoelectric effect or applying non-uniform actuation potential along longitudinal direction (e.g., in using segmented piezoelectric actuator layers), these electric field components cannot be neglected and should be considered in the analysis.

To overcome the limitations of the available theories, in the present study a coupled refined high-order global-local laminate theory is developed based on the double superposition hypothesis for the static analysis of shear-mode piezoelectric sandwich composite beams. In the presence of non-zero longitudinal electric field, the proposed theory not only satisfies the continuity conditions of the transverse shear stresses at the top and the bottom surfaces of the piezoelectric layers, but the non-homogenous shear traction conditions are also exactly satisfied. The boundary conditions of normal tractions are also fulfilled on the upper and the lower surfaces of the beam. Besides, the continuity conditions of the displacement components, transverse normal stresses, and the transverse normal stress gradient at the layer interfaces are satisfied. This novel coupled refined global-local theory is also able to capture the transverse normal strains induced through the piezoelectric layers. In the proposed model, the in-plane displacement component is described by a combination of polynomial and exponential expressions with a layerwise term which contains electrical unknowns. A combination of continuous piecewise fourth-order polynomial with a combination of layerwise components and first order differentiation of electrical unknowns is assumed for the transverse displacement component. As for the electric field component, a quadratic electric potential is considered across the thickness direction of the piezoelectric layers. Considering some novel features, the present model is computationally cost-effective more significantly, and has only one additional independent generalized unknown mechanical parameter compared with FSDT.

Based on the proposed model, a three nodded shear locking-free beam element is employed. The virtual work principle leads to a derivation that could include dynamic analysis. However, in this study only static problems have been considered. Various validation examples of thin and thick beams are examined using a written computer code whose algorithm is based on the present model. The obtained numerical results exhibit a good agreement with the 3D exact piezoelasticity solutions and the coupled 3D finite element (ABAQUS) results.

\section{Formulation of the theory}

\subsection{Geometry and the coordinate system}

In this study, the considered piezoelectric beam is considered to be prismatic with a rectangular uniform cross section. The rectangular cross section has a height of $h$ and width of $b$.This beam is made of $N$ layers with different linearly elastic materials. Each of these layers may be shear-mode piezoelectric. The geometric parameters of the considered laminated beam and the Cartesian coordinate system ( $x, y, z)$ are shown in Fig. 1. Moreover, in order to use the local components in the displacement equations, a transverse local coordinate system is selected for the present piezoelectric beam model (Fig. 1). 


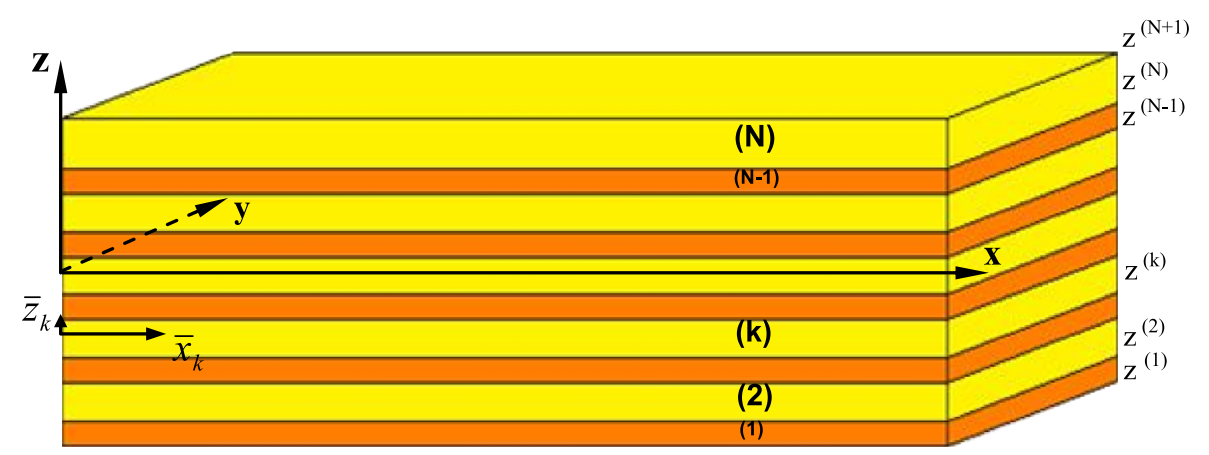

Fig. 1. Geometric parameters, global and local coordinate system of the present laminated beam.

\subsection{Constitutive coupled equations}

In the present model, the piezoelectric and non-piezoelectric materials are assumed to be orthotropic and homogeneous along the span of the beam. It is also assumed that the poling direction of the piezoelectric layers is in the $x$ direction. Based on the previous assumptions, the 3D linear constitutive equations for the $k$-th layer, in its global material coordinate system, can be written as

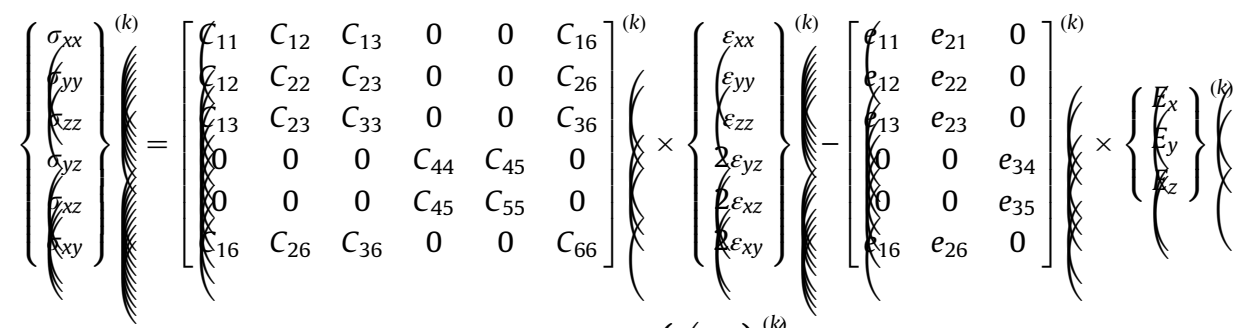

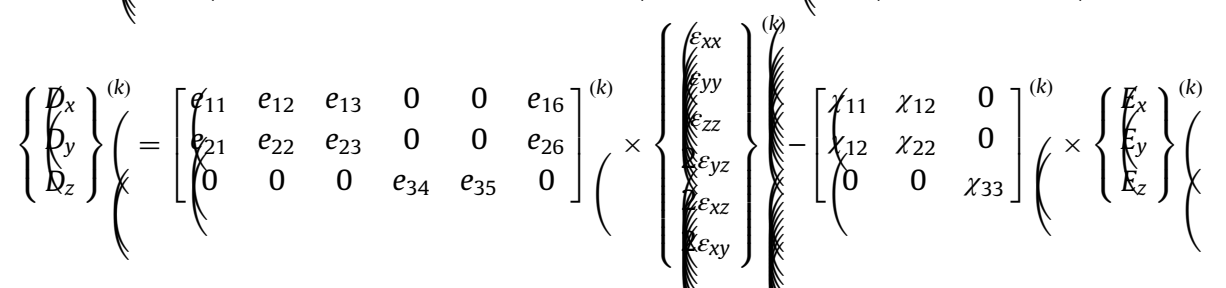

where $\sigma_{i j}, \varepsilon_{i j}$ and $E_{i}$ denote the stress tensor, the infinitesinal strin tensor and the electric field components respectively. $D_{i}$ is the electric displacement vector components, and $c_{k l}, e_{i k}, \chi_{i j}$ are the elastic, piezoelectric and dielectric material constants. For beams with small widths, the following assumptions are made:

$$
\sigma_{y y} \cong 0, \quad \tau_{y z} \cong 0 \quad \tau_{x y} \cong 0, \quad E_{y} \cong 0
$$

Using the conditions (2) and using the static condensation procedure, the 3D linear constitutive relations (1a) and (1b) could be expressed as

$$
\begin{aligned}
\boldsymbol{\sigma} & =\overline{\boldsymbol{C}} \boldsymbol{\varepsilon}-\overline{\boldsymbol{e}}^{\boldsymbol{T}} \boldsymbol{E} \\
\boldsymbol{D} & =\overline{\boldsymbol{e}} \boldsymbol{\varepsilon}+\bar{\chi} E
\end{aligned}
$$

where

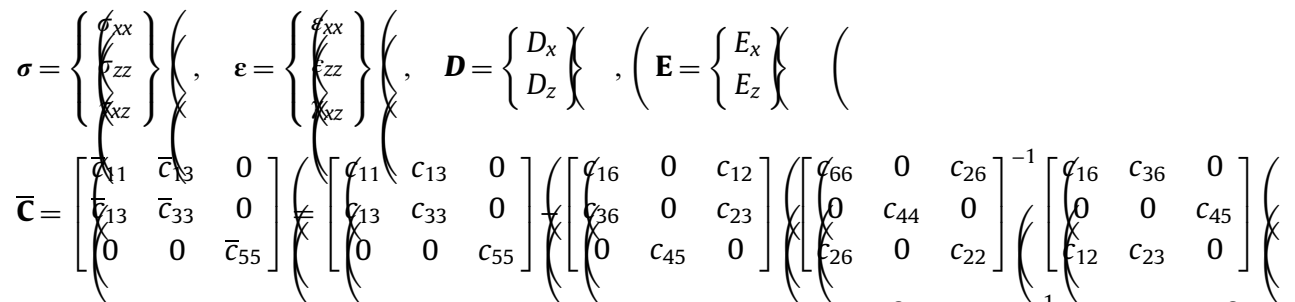

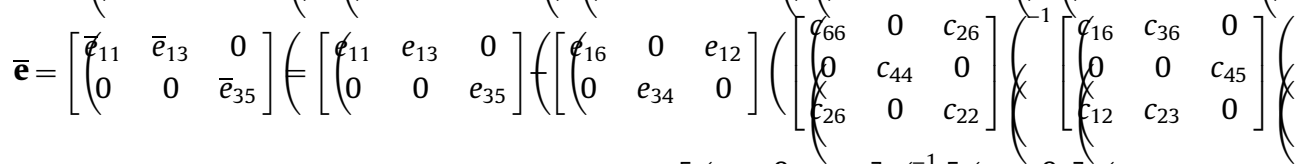

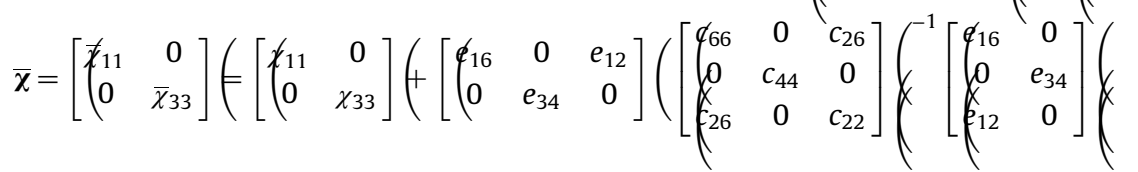


For the beams with infinite dimension along $y$ direction, a state of plane strain exists, that is $\varepsilon_{y y} \cong 0, \gamma_{y z} \cong 0, \gamma_{x y} \cong 0$ and $E_{y} \cong 0$. For this case, the $3 \mathrm{D}$ linear constitutive relations (1a) and (1b) could be expressed as

$$
\begin{aligned}
\boldsymbol{\sigma} & =\overline{\boldsymbol{C}} \boldsymbol{\varepsilon}-\overline{\boldsymbol{e}}^{\boldsymbol{T}} \boldsymbol{E} \\
\boldsymbol{D} & =\overline{\boldsymbol{e}} \boldsymbol{\varepsilon}+\bar{\chi} E
\end{aligned}
$$

where

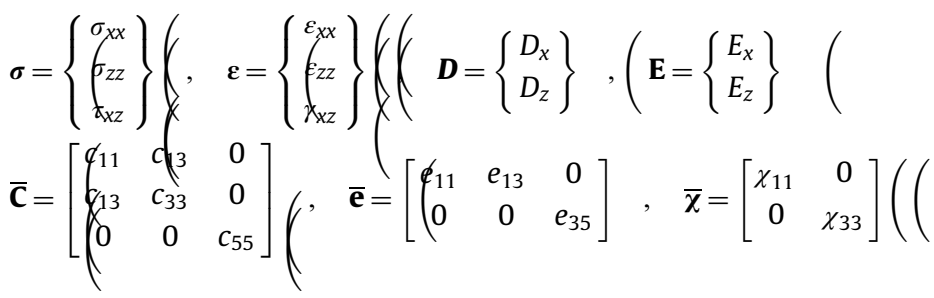

\subsection{Approximation of the electric potential}

In this study the following quadratic electric potential has been considered across the thickness direction of the $k$-th shear piezoelectric layer:

$$
\phi^{k}(x, y, z)=L_{1}^{k}(z) \phi_{b}^{k}(x)+L_{3}^{k}(z) \phi_{c}^{k}(x)+L_{2}^{k}(z) \phi_{t}^{k}(x)
$$

where $\phi_{b}^{k}(x), \phi_{c}^{k}(x)$ and $\phi_{t}^{k}(x)$ denote the electric potential at the bottom, the center and the top of the $k$-th piezoelectric layer, respectively. $L_{j}^{k}(z)$ are the interpolation functions as follows:

$$
L_{1}^{k}(z)=-\frac{1}{2} \bar{z}_{k}\left(1-\bar{z}_{k}\right) \quad, \quad L_{3}^{k}(z)=\left(1+\bar{z}_{k}\right)\left(1-\bar{z}_{k}\right) \quad, \quad L_{2}^{k}(z)=\frac{1}{2} \bar{z}_{k}\left(1+\bar{z}_{k}\right)
$$

where $\bar{z}_{k}=a_{k} z-b_{k}, a_{k}=2 / z_{k+1}-z_{k}, b_{k}=z_{k+1}+z_{k} / z_{k+1}-z_{k}$. The electric field components can be related to the electric potential using the following relations:

$$
\mathbf{E}^{k}=\left[\begin{array}{c}
E_{x}^{k} \\
E_{z}^{k}
\end{array}\right]=\left\{\begin{array}{l}
\frac{\partial \phi^{k}}{\partial x} \\
\frac{\partial \phi^{k}}{\partial z}
\end{array}\right\}=-\left[\begin{array}{ccc}
f_{1}^{k}(z) \frac{d}{d x} & L_{3}^{k}(z) \frac{d}{d x} & L_{2}^{k}(z) \frac{d}{d x} \\
\frac{d L_{1}^{k}}{d z} & \frac{d L_{2}^{k}}{d z} & \frac{d L^{k}}{d z}
\end{array}\right]\left\{\begin{array}{c}
\phi_{b}^{k} \\
k \\
k \\
k \\
k \\
t
\end{array}\right\}
$$

\subsection{Approximation of the displacement and the strain fields}

In the present study, the following refined high-order global-local displacement field is employed $(k=1,2,3, \ldots, N)$ :

$$
\begin{aligned}
& u^{k}(x, y, z)=u_{0}(x)-z w_{0}(x)_{, x}+z \cdot \exp \left(f(z / h)^{2}\right)\left[\theta(x)+w_{0}(x)_{, x}\right]+\bar{u}_{L}^{k}(x, z)+\hat{u}_{L}^{k}(x, z) \\
& w^{k}(x, y, z)=w_{0}(x)+z w_{1}(x)+z^{2} w_{2}(x)+z^{3} w_{3}(x)+z^{4} w_{4}(x)+\sum_{j=1}^{k-1} \psi_{j}(x, t)\left(z-z_{j+1}\right) H\left(z-z_{j+1}\right)+\sum_{j=1}^{k-1} \Psi_{j}(x, t)\left(z-z_{j+1}\right)^{2} H\left(z-z_{j+1}\right)
\end{aligned}
$$

where $H(z)$ is the Heaviside's function. The functions $u^{k}(x, y, z)$ and $w^{k}(x, y, z)$ represent the horizontal (in-plane) and vertical (transverse) displacement components, respectively. The $u_{0}(x), w_{0}(x), w_{1}(x), w_{2}(x), w_{3}(x)$ and $w_{4}(x)$ are global displacement parameters that are independent of $\mathrm{z}$ coordinate. $\theta(x)$ denotes the shear-bending rotation around the $y$ axis. $\psi_{j}(x)$ and $\psi_{j}(x)$ are functions to be determined to fulfill the transverse normal stress and stress gradient continuity conditions at the laminate interfaces. The local components $\bar{u}_{L}^{k}(x, z)$ and $\hat{u}_{L}^{k}(x, z)$ can be chosen based on the layerwise variations concept. Therefore, if they are chosen as combinations of the Legendre polynomial to simplify the numerical integration process, one may write:

$$
\begin{aligned}
& \bar{u}_{L}^{k}(x, z)=\bar{z}_{k} u_{1}^{k}(x)+\frac{3 \bar{z}_{k}^{2}-1}{2} u_{2}^{k}(x) \\
& \hat{u}_{L}^{k}(x, z)=\frac{5 \bar{z}_{k}^{3}-3 \bar{z}_{k}}{2} u_{3}^{k}(x)
\end{aligned}
$$

A graphical illustration of the above high-order global-local displacement field (Eq. 7) is shown in Fig. 2. [43]

Local variations of the transverse displacement component are represented by the two summations in Eq. (7b), employing a discretelayer concept. The continuity conditions of the displacement components at the laminate interfaces should be satisfied. Due to using a layerwise description for the local term, the transverse displacement component satisfies the kinematic continuity condition automatically. By imposing the continuity conditions on the in-plane displacement, the following two equations are resulted:

$$
\begin{aligned}
& \bar{u}_{L}^{k}\left(x, z_{k}\right)=\bar{u}_{L}^{k-1}\left(x, z_{k}\right) \quad, \quad k=2,3, \ldots, N \\
& \hat{u}_{L}^{k}\left(x, z_{k}\right)=\hat{u}_{L}^{k-1}\left(x, z_{k}\right) \quad, \quad k=2,3, \ldots, N
\end{aligned}
$$

Eq. (9) leads to the following equations:

$$
u_{2}^{k}=u_{1}^{k}+u_{1}^{k-1}+u_{2}^{k-1}
$$



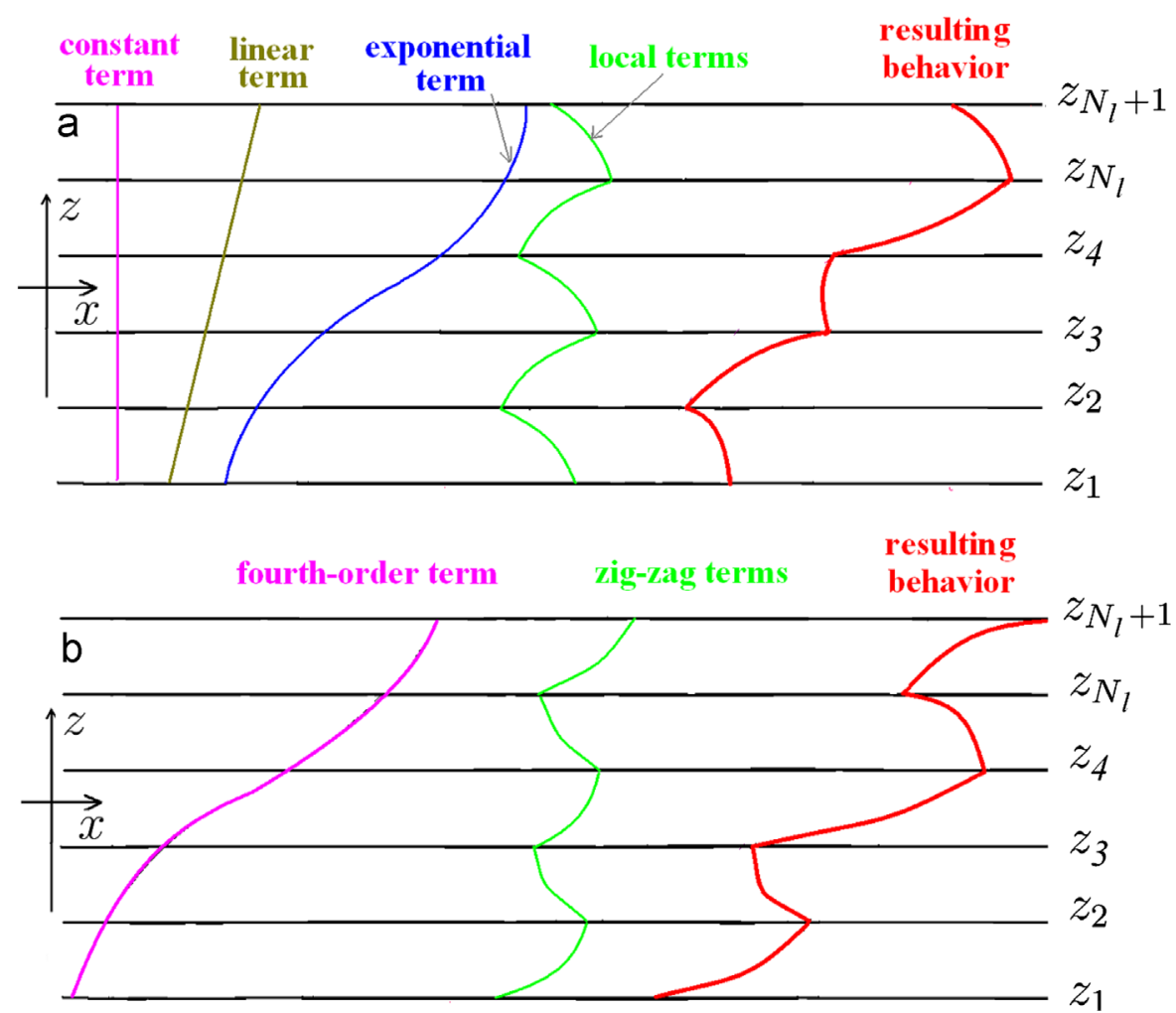

Fig. 2. A graphical illustration for the refined high-order global-local displacement field: (a) in-plane displacement and (b) transverse displacement.

$$
u_{3}^{k}=(-1)^{k-1} u_{3}^{1}
$$

The transverse shear stress of the $k$-th layer may be determined from the following equation:

$$
\begin{aligned}
\tau_{x z}^{k}(x, z)= & G_{k}\left(\oint \operatorname{xp}\left(f 2(z / h)^{2}\right)-4\left(\frac{z}{h}\right)^{2} \exp \left(f 2(z / h)^{2}\right)\right)\left(\phi(x)+w_{0}(x)_{, x}\right)+G_{k} a_{k} u_{1}^{k}+3 G_{k} \bar{z}_{k} a_{k} u_{2}^{k} \\
& +G_{k}\left(\left(-\frac{3}{2} a_{k}+\frac{15}{2} a_{k} \bar{z}_{k}^{2}\right) u_{3}^{k}+e_{35}^{k} \frac{d L_{1}^{k}}{d z} \phi_{b}^{k}+e_{35}^{k} \frac{d L_{3}^{k}}{d z} \phi_{c}^{k}+e_{35}^{k} \frac{d L_{2}^{k}}{d z} \phi_{t}^{k}\right.
\end{aligned}
$$

where $G_{k}=C_{55}^{k}$ is the transverse shear modulus of the $k$-th layer. Note that the simplifying assumption $w^{k}(x, y, z)=w_{0}(x)_{\otimes}$ is used in the computation of the transverse shear stress only to avoid the computational complexity. By imposing the transverse shear stress continuity condition at the mutual interfaces of the adjacent layers $\left(\tau_{x z}^{k}\left(x, z_{k}\right)=\tau_{x z}^{k-1}\left(x, z_{k}\right)\right)$, the following recursive condition is obtained: $(k=2,3, \ldots, N)$

$$
\begin{aligned}
G_{k}( & \left.\exp \left(f 2\left(z_{k} / h\right)^{2}\right)-4\left(\frac{z_{k}}{h}\right)^{2} \exp \left(f 2\left(z_{k} / h\right)^{2}\right)\right)\left(\phi(x)+w_{0}(x)_{, x}\right)+G_{k} a_{k} u_{1}^{k}-3 G_{k} a_{k} u_{2}^{k}+6 G_{k} a_{k} u_{3}^{k} \\
& +e_{35}^{k} \frac{d L_{1}^{k}}{d z}\left(z_{k}\right) \phi_{b}^{k}+e_{35}^{k} \frac{d L_{3}^{k}}{d z}\left(z_{k}\right) \phi_{c}^{k}+e_{35}^{k} \frac{d L_{2}^{k}}{d z}\left(z_{k}\right) \phi_{t}^{k} \\
= & G_{k-1}\left(\oint \operatorname{xp}\left(f\left(z_{k} / h\right)^{2}\right)-4\left(\frac{z_{k}}{h}\right)^{2} \exp \left(f\left(z_{k} / h\right)^{2}\right)\right)\left(g(x)+w_{0}(x)_{, x}\right)+G_{k-1} a_{k-1} u_{1}^{k-1}-3 G_{k-1} a_{k-1} u_{2}^{k-1} \\
& +6 G_{k-1} a_{k-1} u_{3}^{k-1}+e_{35}^{k-1} \frac{d L_{1}^{k-1}}{d z}\left(z_{k}\right) \phi_{b}^{k-1}+e_{35}^{k-1} \frac{d L_{3}^{k-1}}{d z}\left(z_{k}\right) \phi_{c}^{k-1}+e_{35}^{k-1} \frac{d L_{2}^{k-1}}{d z}\left(z_{k}\right) \phi_{t}^{k-1}
\end{aligned}
$$

Furthermore, the boundary conditions of the prescribed values of the shear tractions (that are generally non-zero values) on the top and the bottom surfaces of the shear piezoelectric laminated/sandwich beam should be satisfied. Thus, the following two boundary conditions are obtained:

$$
\begin{aligned}
& \tau_{x z}^{1}\left(z=-\frac{h}{2}\right)=G_{1} a_{1} u_{1}^{1}-3 G_{1} a_{1} u_{2}^{1}+6 G_{1} a_{1} u_{3}^{1}+e_{35}^{1} \frac{d L_{1}^{1}}{d z}\left(-\frac{h}{2}\right) \phi_{b}^{1}+e_{35}^{1} \frac{d L_{3}^{1}}{d z}\left(-\frac{h}{2}\right) \phi_{c}^{1}+e_{35}^{1} \frac{d L_{2}^{1}}{d z}\left(-\frac{h}{2}\right) \phi_{t}^{1}=X^{-}(x) \\
& \tau_{x z}^{N}\left(z=\frac{h}{2}\right)=G_{N} a_{N} u_{1}^{N}+3 G_{N} a_{N} u_{2}^{N}+6 G_{N} a_{N} u_{3}^{N}+e_{35}^{N} \frac{d L_{1}^{N}}{d z}\left(\frac{h}{2}\right) \phi_{b}^{N}+e_{35}^{N} \frac{d L_{3}^{N}}{d z}\left(\frac{h}{2}\right) \phi_{c}^{N}+e_{35}^{N} \frac{d L_{2}^{N}}{d z}\left(\frac{h}{2}\right) \phi_{t}^{N}=X^{+}(x)
\end{aligned}
$$

where $X^{-}(x)$ and $X^{+}(x)$ are the prescribed shear tractions of the bottom and the top surfaces of the beam, respectively. By using of Eqs. (10a) and (12), the following recursive equations are obtained:

$$
\begin{aligned}
& u_{1}^{k}=F_{1}^{k}\left(\theta+w_{0, x}\right)+F_{2}^{k} u_{1}^{k-1}+F_{3}^{k} u_{2}^{k-1}+F_{4}^{k} u_{3}^{k}+F_{5}^{k} \varphi_{b}^{k}+F_{6}^{k} \phi_{c}^{k}+F_{7}^{k} \phi_{t}^{k}+F_{8}^{k} \phi_{b}^{k-1}+F_{9}^{k} \phi_{c}^{k-1}+F_{10}^{k} \phi_{t}^{k-1} \\
& u_{2}^{k}=H_{1}^{k}\left(\theta+w_{0, x}\right)+H_{2}^{k} u_{1}^{k-1}+H_{3}^{k} u_{2}^{k-1}+H_{4}^{k} u_{3}^{k}+H_{5}^{k} \phi_{b}^{k}+H_{6}^{k} \phi_{c}^{k}+H_{7}^{k} \phi_{t}^{k}+H_{8}^{k} \phi_{b}^{k-1}+H_{9}^{k} \phi_{c}^{k-1}+H_{10}^{k} \phi_{t}^{k-1}
\end{aligned}
$$


where the coefficients $F_{j}^{k}$ and $H_{j}^{k}$ are $(j=1,2, \ldots, 10)$ :

$$
\begin{aligned}
& F_{1}^{k}=\frac{\left(\exp \left(-2\left(z_{k} / h\right)^{2}\right)-4\left(z_{k} / h\right)^{2} \exp \left(-2\left(z_{k} / h\right)^{2}\right)\right)\left(G_{k}-G_{k-1}\right)}{2 G_{k} a_{k}} \quad, \quad H_{1}^{k}=F_{1}^{k} \\
& F_{2}^{k}=\frac{\left(-G_{k-1} a_{k-1}-3 G_{k} a_{k}\right)}{2 G_{k} a_{k}} \quad, \quad H_{2}^{k}=\frac{\left(-G_{k-1} a_{k-1}-G_{k} a_{k}\right)}{2 G_{k} a_{k}} \\
& F_{3}^{k}=\frac{\left(-3 G_{k-1} a_{k-1}-3 G_{k} a_{k}\right)}{2 G_{k} a_{k}} \quad, \quad H_{3}^{k}=\frac{\left(-3 G_{k-1} a_{k-1}-G_{k} a_{k}\right)}{2 G_{k} a_{k}} \\
& F_{4}^{k}=\frac{\left(6 G_{k-1} a_{k-1}+6 G_{k} a_{k}\right)}{2 G_{k} a_{k}} \quad, \quad H_{4}^{k}=F_{4}^{k} \\
& F_{5}^{k}=\frac{e_{35}^{k}\left(d L_{1}^{k} / d z\right)\left(z_{k}\right)}{2 G_{k} a_{k}}, \quad H_{5}^{k}=F_{5}^{k} \\
& F_{6}^{k}=\frac{\left.e_{35}^{k}(d L / k) / d z\right)\left(z_{k}\right)}{2 G_{k} a_{k}} \quad, \quad H_{6}^{k}=F_{6}^{k} \\
& F_{7}^{k}=\frac{e_{35}^{k}\left(d L_{2}^{k} / d z\right)\left(z_{k}\right)}{2 G_{k} a_{k}}, \quad H_{7}^{k}=F_{7}^{k} \\
& F_{8}^{k}=-\frac{e_{35}^{k-1}\left(d L_{1}^{k-1} / d z\right)\left(z_{k}\right)}{2 G_{k} a_{k}}, \quad H_{8}^{k}=F_{8}^{k} \\
& F_{9}^{k}=-\frac{e_{35}^{k-1}\left(d L_{2}^{k-1} / d z\right)\left(z_{k}\right)}{2 G_{k} a_{k}}, \quad H_{9}^{k}=F_{9}^{k} \\
& F_{10}^{k}=\frac{e_{35}^{k}\left(d L_{2}^{k-1} / d z\right)\left(Z_{k}\right)}{2 G_{k} a_{k}} \quad, \quad H_{10}^{k}=F_{10}^{k}
\end{aligned}
$$

Substituting Eq. (10b) into Eq. (13a) yields:

$$
u_{3}^{k}=\frac{(-1)^{k-1}}{6 G_{1} a_{1}} X^{-}+\frac{(-1)^{k}}{6} u_{1}^{1}+\frac{(-1)^{k-1}}{2} u_{2}^{1}+(-1)^{k} \frac{e_{35}^{1}\left(d L_{1}^{1} / d z\right)(-h / 2)}{6 G_{1} a_{1}} \phi_{b}^{1}+(-1)^{k} \frac{e_{35}^{1}\left(d L_{3}^{1} / d z\right)\left(-\frac{h}{2}\right)}{6 G_{1} a_{1}} \phi_{c}^{1}+(-1)^{k} \frac{e_{35}^{1}\left(d L_{2}^{1} / d z\right)(-h / 2)}{6 G_{1} a_{1}} \phi_{t}^{1}
$$

The above equation expresses $u_{3}^{k}$ in terms of $u_{1}^{1}, u_{2}^{1}, X^{-}, \phi_{b}^{1}, \phi_{c}^{1}$ and $\phi_{t}^{1}$. Substituting Eq. (16) into the recursive Eq. (14) related $u_{1}^{k}$ and $u_{2}^{k}$ to $u_{1}^{1}, u_{2}^{1},\left(\theta+w_{0, x}\right), X^{-}, \phi_{b}^{i}, \phi_{c}^{i}$ and $\phi_{t}^{i}$ where $i-1,2, \ldots, P N$ and $P N$ denotes the number of shear piezoelectric layers. After calculating $u_{1}^{N}, u_{2}^{N}$ and $u_{3}^{N}$ from the recursive Eqs. (14) and (16), respectively and substituting them into Eq. (13b), $u_{2}^{1}$ can be eliminated. Thus, Eqs. (14) and (16) can be rewritten as:

$$
\begin{aligned}
& u_{1}^{k}(x)=\alpha_{1}^{k}\left(q(x)+w_{0}(x)_{, x}\right)\left(\beta_{1}^{k} u_{1}^{1}(x)+\delta_{1}^{k} X^{+}(x)+\lambda_{1}^{k} X^{-}(x)+\sum_{i=1}^{P N}\left(q_{1 i}^{k} \phi_{b}^{i}(x)+\mu_{1 i}^{k} \phi_{c}^{i}(x)+\vartheta_{1 i}^{k} \phi_{t}^{i}(x)\right)\right. \\
& u_{2}^{k}(x)=\alpha_{2}^{k}\left(q(x)+w_{0}(x)_{, x}\right)\left(\beta_{2}^{k} u_{1}^{1}(x)+\delta_{2}^{k} X^{+}(x)+\lambda_{2}^{k} X^{-}(x)+\sum_{i=1}^{P N}\left(q_{2 i}^{k} \phi_{b}^{i}(x)+\mu_{2 i}^{k} \phi_{c}^{i}(x)+\vartheta_{2 i}^{k} \phi_{t}^{i}(x)\right)\right. \\
& u_{3}^{k}(x)=\alpha_{3}^{k}\left(q(x)+w_{0}(x)_{, x}\right) f\left(\beta_{3}^{k} u_{1}^{1}(x)+\delta_{3}^{k} X^{+}(x)+\lambda_{3}^{k} X^{-}(x)+\sum_{i=1}^{P N}\left(q_{3 i}^{k} \phi_{b}^{i}(x)+\mu_{3 i}^{k} \phi_{c}^{i}(x)+\vartheta_{3 i}^{k} \phi_{t}^{i}(x)\right)\right.
\end{aligned}
$$

where coefficients $\alpha_{1}^{k}, \alpha_{2}^{k}, \alpha_{3}^{k}, \beta_{1}^{k}, \beta_{2}^{k}, \beta_{3}^{k}, \delta_{1}^{k}, \delta_{2}^{k}, \delta_{3}^{k}, \lambda_{1}^{k}, \lambda_{2}^{k}, \lambda_{3}^{k}, \kappa_{1 i}^{k}, \kappa_{2 i}^{k}, \kappa_{3 i}^{k}, \mu_{1 i}^{k}, \mu_{2 i}^{k}, \mu_{3 i}^{k}, \vartheta_{1 i}^{k}, \vartheta_{2 i}^{k}, \vartheta_{3 i}^{k}$ are obtained from the procedure described above. These coefficients are only dependent on the material properties and the global coordinates of the layers.

The transverse normal stress and stress gradient in the $k$-th layer are determined from the following equations:

$$
\begin{aligned}
\sigma_{z z}^{k}= & C_{13}^{k}\left(u_{0, x}-z w_{0, x x}+J(z)\left(\theta_{, x}+w_{0, x x}\right)+T(z) u_{1, x}^{1}+P(z)\left(X^{+}\right)_{, x}+R(z)\left(X^{-}\right)_{, x}+\sum_{i=1}^{P N}\left(O_{1 i}^{\text {elec }}(z)\left(\phi_{b}^{i}\right)_{x}+O_{2 i}^{\text {elec }}(z)\left(\phi_{c}^{i}\right)_{, x}+O_{3 i}^{\text {elec }}(z)\left(\phi_{t}^{i}\right)_{x}\right)\right) \\
& +C_{33}^{k}\left(w_{1}+2 z w_{2}+3 z^{2} w_{3}+4 z^{3} w_{4}+\sum_{j=1}^{k-1} \psi_{j} H\left(z-z_{j+1}\right)+2 \sum_{j=1}^{k-1} \Psi_{j}\left(z-z_{j+1}\right) H\left(z-z_{j+1}\right)\right) \\
& +e_{13}^{k}\left(L_{1}^{k}(z) \frac{d \phi_{b}^{k}}{d x}+L_{3}^{k}(z) \frac{d \phi_{c}^{k}}{d x}+L_{2}^{k}(z) \frac{d \phi_{t}^{k}}{d x}\right) \\
\sigma_{z z, z}^{k} & =C_{13}^{k}\left(-w_{0, x x}+J(z)_{, z}\left(\theta_{, x}+w_{0, x x}\right)+T(z)_{, z} u_{1, x}^{1}+P(z)_{, z}\left(X^{+}\right)_{, x}+R(z)_{, z}\left(X^{-}\right)+\sum_{i=1}^{P N}\left(O_{1 i}^{\text {elec }}(z)_{, z}\left(\phi_{b}^{i}\right)_{, x}+O_{2 i}^{\text {elec }}(z)_{, z}\left(\varphi_{c}^{i}\right)_{, x}+O_{3 i}^{\text {elec }}(z)_{, z}\left(\phi_{t}^{i}\right)_{, x}\right)\right) \\
& +C_{33}^{k}\left(2 w_{2}+6 z w_{3}+12 z^{2} w_{4}+2 \sum_{j=1}^{k-1} \Psi_{j} H\left(z-z_{j+1}\right)\right)+e_{13}^{k}\left(L_{1}^{k}(z)_{, z} \frac{d \phi_{b}^{k}}{d x}+L_{3}^{k}(z)_{, z} \frac{d \phi_{c}^{k}}{d x}+L_{2}^{k}(z), z \frac{d \phi_{t}^{k}}{d x}\right)
\end{aligned}
$$

where

$$
\begin{aligned}
& \left.\left.\left.J(z)=z \cdot \exp \left(-2(z / h)^{2}\right)+\sum_{k=1}^{N} \bar{z}_{k} \alpha_{1}^{k}+\frac{3 \bar{z}_{k}^{2}-1}{2}\right) \phi_{2}^{k}+\frac{5 \bar{z}_{k}^{3}-3 \bar{z}_{k}}{2}\right) \alpha_{3}^{k}\right)\left(\times\left(H\left(z-z_{k}\right)-H\left(z-z_{k+1}\right)\right)\right. \\
& \left.\left.\left.T(z)=\sum_{k=1}^{N} \bar{z}_{k} \beta_{1}^{k}+\frac{3 \bar{z}_{k}^{2}-1}{2}\right) k_{2}^{k}+\frac{5 \bar{z}_{k}^{3}-3 \bar{z}_{k}}{2}\right) \phi_{(k}^{k}\right)\left(\times\left(H\left(z-z_{k}\right)-H\left(z-z_{k+1}\right)\right)\right. \\
& \left.\left.\left.P(z)=\sum_{k=1}^{N} \bar{z}_{k} \delta_{1}^{k}+\frac{3 \bar{z}_{k}^{2}-1}{2}\right) \phi_{2}^{k}+\frac{5 \bar{z}_{k}^{3}-3 \bar{z}_{k}}{2}\right) d_{(k}\right)\left(\times\left(H\left(z-z_{k}\right)-H\left(z-z_{k+1}\right)\right)\right.
\end{aligned}
$$




$$
\begin{aligned}
& \left.R(z)=\sum_{k=1}^{N} \bar{z}_{k} \lambda_{1}^{k}+\frac{3 \bar{z}_{k}^{2}-\check{C}}{2}\right)\left(\begin{array}{l}
k \\
2
\end{array}+\frac{5 \bar{z}_{k}^{3}-3 \bar{z}_{k}}{2}\right)\left(\lambda_{3}^{k}\right)\left(\times\left(H\left(z-z_{k}\right)-H\left(z-z_{k+1}\right)\right)\right. \\
& \left.\left.O_{1 i}^{\text {elec }}(z)=\sum_{k=1}^{N} \bar{z}_{k} \kappa_{1 i}^{k}+\frac{3 \bar{z}_{k}^{2}-1}{2}\right),\left(k_{2 i}^{k}+\frac{5 \bar{z}_{k}^{3}-3 \bar{z}_{k}}{2}\right) \kappa_{i i}\right)\left(\times\left(H\left(z-z_{k}\right)-H\left(z-z_{k+1}\right)\right)\right. \\
& \left.\left.O_{2 i}^{\text {elec }}(z)=\sum_{k=1}^{N} \bar{z}_{k} \mu_{1 i}^{k}+\frac{3 \bar{z}_{k}^{2}-1}{2}\right) \mu_{2 i}^{k}+\frac{5 \bar{z}_{k}^{3}-3 \bar{z}_{k}}{2}\right)\left(\mu_{3 i}^{k}\right) \times\left(H\left(z-z_{k}\right)-H\left(z-z_{k+1}\right)\right) \\
& \left.\left.O_{3 i}^{\text {elec }}(z)=\sum_{k=1}^{N} \bar{z}_{k} \vartheta_{1 i}^{k}+\frac{3 \bar{z}_{k}^{2}-1}{2}\right)\left(\psi_{2 i}^{k}+\frac{5 \bar{z}_{k}^{3}-3 \bar{z}_{k}}{2}\right) \vartheta \vartheta_{i}\right)\left(\times\left(H\left(z-z_{k}\right)-H\left(z-z_{k+1}\right)\right)\right.
\end{aligned}
$$

Continuity of $\sigma_{z z}$ and $\sigma_{z z, z}$ must be fulfilled at $N-1$ interfaces:

$$
\begin{aligned}
& \sigma_{z z}^{k+1}\left(z_{k+1}\right)=\sigma_{z z}^{k}\left(z_{k+1}\right) \quad, \quad k=1,2, \ldots, N-1 \\
& \sigma_{z z, z}^{k+1}\left(z_{k+1}\right)=\sigma_{z z, z}^{k}\left(z_{k+1}\right) \quad, \quad k=1,2, \ldots, N-1
\end{aligned}
$$

From the recursive Eqs. (20), $\psi_{k}(x)$ and $\Psi_{k}(x)$ will have the following forms $(k=1,2, \ldots, N-1)$ :

$$
\begin{aligned}
\Psi_{k}= & A_{1}^{k} w_{0, x x}+A_{2}^{k} w_{2}+A_{3}^{k} w_{3}+A_{4}^{k} w_{4}+A_{5}^{k} \theta_{, x}+A_{6}^{k}\left(u_{1}^{1}\right)_{, x}+A_{7}^{k}\left(X^{+}\right)_{, x}+A_{8}^{k}\left(X^{-}\right)_{, x} \\
& +\sum_{i=1}^{P N}\left(A_{6+3 i}^{k}\left(\phi_{b}^{i}\right)_{, x}+A_{7+3 i}^{k}\left(\phi_{c}^{i}\right)_{, x}+A_{8+3 i}^{k}\left(\phi_{t}^{i}\right)_{, x}\right) \\
\psi_{k}= & B_{1}^{k} w_{0, x x}+B_{2}^{k} w_{1}+B_{3}^{k} w_{2}+B_{4}^{k} w_{3}+B_{5}^{k} w_{4}+B_{6}^{k}\left(u_{0}\right)_{, x}+B_{7}^{k} \theta_{, x}+B_{8}^{k}\left(u_{1}^{1}\right)_{, x}+B_{9}^{k}\left(X^{+}\right)_{, x}+B_{10}^{k}\left(X^{-}\right)_{, x} \\
& +\sum_{i=1}^{P N}\left(B_{8+3 i}^{k}\left(\phi_{b}^{i}\right)_{, x}+B_{9+3 i}^{k}\left(\phi_{c}^{i}\right)_{, x}+B_{10+3 i}^{k}\left(\phi_{t}^{i}\right)_{, x}\right)
\end{aligned}
$$

Moreover, the boundary conditions of the transverse normal stress and transverse normal stress gradient on the upper and lower faces should be satisfied:

$$
\begin{aligned}
& \sigma_{z z}^{1}\left(z_{1}\right)=Z^{-}(x), \sigma_{z z}^{N}\left(z_{N+1}\right)=Z^{+}(x) \\
& \sigma_{z z, z}^{1}\left(z_{1}\right)=\sigma_{z z, z}^{N}\left(z_{N+1}\right)=0
\end{aligned}
$$

where $Z^{+}(x)$ and $Z^{-}(x)$ are the distributed lateral loads acting on the bottom and the top surfaces of the beam, respectively. From Eqs. (22), the unknowns $w_{1}, w_{2}, w_{3}$ and $w_{4}$ can be expressed in terms of $u_{0, x}, w_{0, x x}, \theta_{, x},\left(u_{1}^{1}\right)_{, x},\left(X^{+}\right)_{, x},\left(X^{-}\right)_{, x}, Z^{+}, Z^{-},\left(\phi_{b}^{i}\right)_{, x},\left(\phi_{c}^{i}\right)_{, x}$ and $\left(\phi_{t}^{i}\right)_{, x}(i=1,2, \ldots, P N)$ :

$$
\begin{aligned}
w_{1}(x)= & \Theta_{1}^{1}\left(u_{0}\right)_{, x}+\Theta_{2}^{1} w_{0, x x}+\Theta_{3}^{1} \theta_{, x}+\Theta_{4}^{1}\left(u_{1}^{1}\right)_{, x}+\Theta_{5}^{1}\left(X^{+}\right)_{, x}+\Theta_{6}^{1}\left(X^{-}\right)_{, x}+\Theta_{7}^{1} Z^{+}+\Theta_{8}^{1} Z^{-} \\
& +\sum_{i=1}^{P N}\left(\Theta_{6+3 i}^{1}\left(\phi_{b}^{i}\right)_{, x}+\Theta_{7+3 i}^{1}\left(\varphi_{c}^{i}\right)_{, x}+\Theta_{8+3 i}^{1}\left(\phi_{t}^{i}\right)_{, x}\right) \\
w_{2}(x)= & \Theta_{1}^{2}\left(u_{0}\right)_{, x}+\Theta_{2}^{2} w_{0, x x}+\Theta_{3}^{2} \theta_{, x}+\Theta_{4}^{2}\left(u_{1}^{1}\right)_{, x}+\Theta_{5}^{2}\left(X^{+}\right)_{, x}+\Theta_{6}^{2}\left(X^{-}\right)_{, x}+\Theta_{7}^{2} Z^{+}+\Theta_{8}^{2} Z^{-} \\
& +\sum_{i=1}^{P N}\left(\Theta_{6+3 i}^{2}\left(\phi_{b}^{i}\right)_{, x}+\Theta_{7+3 i}^{2}\left(\phi_{c}^{i}\right)_{, x}+\Theta_{8+3 i}^{2}\left(\phi_{t}^{i}\right)_{, x}\right) \\
w_{3}(x)= & \Theta_{1}^{3}\left(u_{0}\right)_{, x}+\Theta_{2}^{3} w_{0, x x}+\Theta_{3}^{3} \theta_{, x}+\Theta_{4}^{3}\left(u_{1}^{1}\right)_{, x}+\Theta_{5}^{3}\left(X^{+}\right)_{, x}+\Theta_{6}^{3}\left(X^{-}\right)_{, x}+\Theta_{7}^{3} Z^{+}+\Theta_{8}^{3} Z^{-} \\
& +\sum_{i=1}^{P N}\left(\Theta_{6+3 i}^{3}\left(\phi_{b}^{i}\right)_{, x}+\Theta_{7+3 i}^{3}\left(\phi_{c}^{i}\right)_{, x}+\Theta_{8+3 i}^{3}\left(\phi_{t}^{i}\right)_{, x}\right) \\
w_{4}(x)= & \Theta_{1}^{4}\left(u_{0}\right)_{, x}+\Theta_{2}^{4} w_{0, x x}+\Theta_{3}^{4} \theta_{, x}+\Theta_{4}^{4}\left(u_{1}^{1}\right)_{, x}+\Theta_{5}^{4}\left(X^{+}\right)_{, x}+\Theta_{6}^{4}\left(X^{-}\right)_{, x}+\Theta_{7}^{4} Z^{+}+\Theta_{8}^{4} Z^{-} \\
& +\sum_{i=1}^{P N}\left(\Theta_{6+3 i}^{4}\left(\phi_{b}^{i}\right)_{, x}+\Theta_{7+3 i}^{4}\left(\phi_{c}^{i}\right)_{, x}+\Theta_{8+3 i}^{4}\left(\phi_{t}^{i}\right)_{, x}\right)
\end{aligned}
$$

By substituting Eqs. (23) into Eqs. (21), $\psi_{k}(x)$ and $\Psi_{k}(x)$ can be rewritten as follows $(k=1,2, \ldots, N-1)$ :

$$
\begin{aligned}
\Psi_{k}(x)= & C_{1}^{k}\left(u_{0}\right)_{, x}+C_{2}^{k} w_{0, x x}+C_{3}^{k} \theta_{, x}+C_{4}^{k}\left(u_{1}^{1}\right)_{, x}+C_{5}^{k}\left(X^{+}\right)_{, x}+C_{6}^{k}\left(X^{-}\right)_{, x}+C_{7}^{k} Z^{+}+C_{8}^{k} Z^{-} \\
& +\sum_{i=1}^{P N}\left(C_{6+3 i}^{k}\left(\phi_{b}^{i}\right)_{, x}+C_{7+3 i}^{k}\left(\phi_{c}^{i}\right)_{, x}+C_{8+3 i}^{k}\left(\phi_{t}^{i}\right)_{, x}\right) \\
\psi_{k}(x)= & D_{1}^{k}\left(u_{0}\right)_{, x}+D_{2}^{k} w_{0, x x}+D_{3}^{k} \theta_{, x}+D_{4}^{k}\left(u_{1}^{1}\right)_{, x}+D_{5}^{k}\left(X^{+}\right)_{, x}+D_{6}^{k}\left(X^{-}\right)_{, x}+D_{7}^{k} Z^{+}+D_{8}^{k} Z^{-} \\
& +\sum_{i=1}^{P N}\left(D_{6+3 i}^{k}\left(\phi_{b}^{i}\right)_{, x}+D_{7+3 i}^{k}\left(\phi_{c}^{i}\right)_{, x}+D_{8+3 i}^{k}\left(\phi_{t}^{i}\right)_{, x}\right)
\end{aligned}
$$

Finally by satisfying the continuity conditions and the boundary conditions, we have been able to determine all the mechanical unknowns of the displacement fields (Eq. 7) in terms of four independent mechanical unknown parameters $u_{0}, w_{0}, \theta$ and $u_{1}^{1}$ and electrical 
unknowns $\phi_{b}^{i}, \phi_{c}^{i}$ and $\phi_{t}^{i}(i=1,2, \ldots, P N)$. Therefore, the final displacement fields of the proposed coupled refined high-order global-local theory can be written as:

$$
\begin{aligned}
u(x, y, z)= & u_{0}-z w_{0, x}+J(z)\left(\theta+w_{0, x}\right)+T(z) u_{1}^{1}+P(z)\left(X^{+}\right)+R(z)\left(X^{-}\right)+\sum_{i=1}^{P N}\left(O_{1 i}^{\text {elec }}(z) \phi_{b}^{i}+O_{2 i}^{\text {elec }}(z) \phi_{c}^{i}+O_{3 i}^{\text {elec }}(z) \phi_{t}^{i}\right) \\
w(x, y, z)= & w_{0}+\Delta_{1}^{k}(z) u_{0, x}+\Delta_{2}^{k}(z) w_{0, x x}+\Delta_{3}^{k}(z) \theta_{, x}+\Delta_{4}^{k}(z)\left(u_{1}^{1}\right)_{, x}+\Delta_{5}^{k}(z)\left(X^{+}\right)_{, x}+\Delta_{6}^{k}(z)\left(X^{-}\right)_{, x} \\
& +\Delta_{7}^{k}(z) Z^{+}+\Delta_{8}^{k}(z) Z^{-}+\sum_{i=1}^{P N}\left(\Delta_{6+3 i}^{k}\left(\phi_{b}^{i}\right)_{, x}+\Delta_{7+3 i}^{k}\left(\phi_{c}^{i}\right)_{, x}+\Delta_{8+3 i}^{k}\left(\phi_{t}^{i}\right)_{, x}\right)
\end{aligned}
$$

where

$$
\begin{aligned}
& \Delta_{1}^{k}(z)=\Theta_{1}^{1} z+\Theta_{1}^{2} z^{2}+\Theta_{1}^{3} z^{3}+\Theta_{1}^{4} z^{4}+\sum_{j=1}^{k-1} D_{1}^{j}\left(z-z_{j+1}\right) H\left(z-z_{j+1}\right)+\sum_{j=1}^{k-1} C_{1}^{j}\left(z-z_{j+1}\right)^{2} H\left(z-z_{j+1}\right) \\
& \Delta_{2}^{k}(z)=\Theta_{2}^{1} z+\Theta_{2}^{2} z^{2}+\Theta_{2}^{3} z^{3}+\Theta_{2}^{4} z^{4}+\sum_{j=1}^{k-1} D_{2}^{j}\left(z-z_{j+1}\right) H\left(z-z_{j+1}\right)+\sum_{j=1}^{k-1} C_{2}^{j}\left(z-z_{j+1}\right)^{2} H\left(z-z_{j+1}\right) \\
& \Delta_{3}^{k}(z)=\Theta_{3}^{1} z+\Theta_{3}^{2} z^{2}+\Theta_{3}^{3} z^{3}+\Theta_{3}^{4} z^{4}+\sum_{j=1}^{k-1} D_{3}^{j}\left(z-z_{j+1}\right) H\left(z-z_{j+1}\right)+\sum_{j=1}^{k-1} C_{3}^{j}\left(z-z_{j+1}\right)^{2} H\left(z-z_{j+1}\right) \\
& \Delta_{4}^{k}(z)=\Theta_{4}^{1} z+\Theta_{4}^{2} z^{2}+\Theta_{4}^{3} z^{3}+\Theta_{4}^{4} z^{4}+\sum_{j=1}^{k-1} D_{4}^{j}\left(z-z_{j+1}\right) H\left(z-z_{j+1}\right)+\sum_{j=1}^{k-1} C_{4}^{j}\left(z-z_{j+1}\right)^{2} H\left(z-z_{j+1}\right) \\
& \Delta_{5}^{k}(z)=\Theta_{5}^{1} z+\Theta_{5}^{2} z^{2}+\Theta_{5}^{3} z^{3}+\Theta_{5}^{4} z^{4}+\sum_{j=1}^{k-1} D_{5}^{j}\left(z-z_{j+1}\right) H\left(z-z_{j+1}\right)+\sum_{j=1}^{k-1} C_{5}^{j}\left(z-z_{j+1}\right)^{2} H\left(z-z_{j+1}\right) \\
& \Delta_{6}^{k}(z)=\Theta_{6}^{1} z+\Theta_{6}^{2} z^{2}+\Theta_{6}^{3} z^{3}+\Theta_{6}^{4} z^{4}+\sum_{j=1}^{k-1} D_{6}^{j}\left(z-z_{j+1}\right) H\left(z-z_{j+1}\right)+\sum_{j=1}^{k-1} C_{6}^{j}\left(z-z_{j+1}\right)^{2} H\left(z-z_{j+1}\right) \\
& \Delta_{7}^{k}(z)=\Theta_{7}^{1} z+\Theta_{7}^{2} z^{2}+\Theta_{7}^{3} z^{3}+\Theta_{7}^{4} z^{4}+\sum_{j=1}^{k-1} D_{7}^{j}\left(z-z_{j+1}\right) H\left(z-z_{j+1}\right)+\sum_{j=1}^{k-1} C_{7}^{j}\left(z-z_{j+1}\right)^{2} H\left(z-z_{j+1}\right) \\
& \Delta_{8}^{k}(z)=\Theta_{8}^{1} z+\Theta_{8}^{2} z^{2}+\Theta_{8}^{3} z^{3}+\Theta_{8}^{4} z^{4}+\sum_{j=1}^{k-1} D_{8}^{j}\left(z-z_{j+1}\right) H\left(z-z_{j+1}\right)+\sum_{j=1}^{k-1} C_{8}^{j}\left(z-z_{j+1}\right)^{2} H\left(z-z_{j+1}\right) \\
& \Delta_{6+3 i}^{k}(z)=\Theta_{6+3 i}^{1} z+\Theta_{6+3 i}^{2} z^{2}+\Theta_{6+3 i}^{3} z^{3}+\Theta_{6+3 i}^{4} z^{4}+\sum_{j=1}^{k-1} D_{6+3 i}^{j}\left(z-z_{j+1}\right) H\left(z-z_{j+1}\right)+\sum_{j=1}^{k-1} C_{6+3 i}^{j}\left(z-z_{j+1}\right)^{2} H\left(z-z_{j+1}\right) \\
& \Delta_{7+3 i}^{k}(z)=\Theta_{7+3 i}^{1} z+\Theta_{7+3 i}^{2} z^{2}+\Theta_{7+3 i}^{3} z^{3}+\Theta_{7+3 i}^{4} z^{4}+\sum_{j=1}^{k-1} D_{7+3 i}^{j}\left(z-z_{j+1}\right) H\left(z-z_{j+1}\right)+\sum_{j=1}^{k-1} C_{7+3 i}^{j}\left(z-z_{j+1}\right)^{2} H\left(z-z_{j+1}\right) \\
& \Delta_{8+3 i}^{k}(z)=\Theta_{8+3 i}^{1} z+\Theta_{8+3 i}^{2} z^{2}+\Theta_{8+3 i}^{3} z^{3}+\Theta_{8+3 i}^{4} z^{4}+\sum_{j=1}^{k-1} D_{8+3 i}^{j}\left(z-z_{j+1}\right) H\left(z-z_{j+1}\right)+\sum_{j=1}^{k-1} C_{8+3 i}^{j}\left(z-z_{j+1}\right)^{2} H\left(z-z_{j+1}\right)
\end{aligned}
$$

Using Cauchy's definition of the strain tensor, the in-plane, transverse shear and normal strain components may be calculated on the proposed coupled global-local description of the displacement field as:

$$
\begin{aligned}
\varepsilon_{x x}= & u_{0, x}-z w_{0, x x}+J(z)\left(\theta_{, x}+w_{0, x x}\right)+T(z) u_{1, x}^{1}+P(z)\left(X^{+}\right)_{, x}+R(z)\left(X^{-}\right)_{, x}+\sum_{i=1}^{P N}\left(O_{1 i}^{\text {elec }}(z)\left(\phi_{b}^{i}\right)_{, x}+O_{2 i}^{\text {elec }}(z)\left(\phi_{c}^{i}\right)_{, x}+O_{3 i}^{\text {elec }}(z)\left(\phi_{t}^{i}\right)_{, x}\right) \\
\varepsilon_{z z}= & \Delta_{1}^{k}(z)_{, z} u_{0, x}+\Delta_{2}^{k}(z)_{, z} w_{0, x x}+\Delta_{3}^{k}(z)_{, z} \theta_{, x}+\Delta_{4}^{k}(z)_{, z}\left(u_{1}^{1}\right)_{, x}+\Delta_{5}^{k}(z)_{, z}\left(X^{+}\right)_{, x}+\Delta_{6}^{k}(z)_{, z}\left(X^{-}\right)_{, x} \\
& +\Delta_{7}^{k}(z)_{, z} Z^{+}+\Delta_{8}^{k}(z)_{, z} Z^{-}+\sum_{i=1}^{P N}\left(\left(\Delta_{6+3 i}^{k}\right)_{, z}\left(\phi_{b}^{i}\right)_{, x}+\left(\Delta_{7+3 i}^{k}\right)_{, z}\left(\phi_{c}^{i}\right)_{, x}+\left(\Delta_{8+3 i}^{k}\right)_{, z}\left(\phi_{t}^{i}\right)_{, x}\right) \\
\gamma_{x z}= & J(z)_{, z}\left(\theta+w_{0, x}\right)+T(z)_{, z} u_{1}^{1}+P(z)_{, z} X^{+}+R(z)_{, z} X^{-}+\sum_{i=1}^{P N}\left(O_{1 i}^{\text {elec }}(z)_{, z} \phi_{b}^{i}+O_{2 i}^{\text {elec }}(z)_{, z} \phi_{c}^{i}+O_{3 i}^{\text {elec }}(z)_{, z} \phi_{t}^{i}\right)
\end{aligned}
$$

Since the functions $u(x, y, z)$ and $w(x, y, z)$ are coupled in the strain expression, it is expected that the presented coupled refined globallocal theory compensates to some extent for the simplifying assumption $w^{k}(x, z)=w_{0}(x)$ used only in the computation of the transverse shear stress.

\section{Finite element model}

In this section, a finite element representation of the displacement field described by Eq. (25) is introduced using appropriate shape functions and nodal variables. As the highest derivative of $w_{0}$ in the expression of the strain energy is of second-order, this variable was interpolated using $\mathrm{C}^{1}$ - continuous Hermite cubic shape functions. The rotation $\theta$ is interpolated by quadratic Lagrangian shape functions to 
ensure obtaining more accurate results. Furthermore, if an identical order is adopted for the shape functions of both $w_{0, x}$ and $\theta$ parameters in the relevant transverse shear strain components, the shear locking phenomenon may be avoided due to using a consistent displacement field $[32,44]$. Finally, $u_{0}, u_{1}^{1}, X^{+}, X^{-}, Z^{+}, Z^{-}, \phi_{b}^{i}, \phi_{c}^{i}$ and $\phi_{t}^{i}$ may be interpolated using Lagrangian quadratic shape functions. The proposed piezoelectric beam element along with its nodal degrees of freedom are shown in Fig. 3. As shown in this figure, the beam element has three nodes with a variable number of electric potential degrees of freedom at each node.

Based on Eqs. (4), (6), (25), (27) and the nodal degrees of freedom, the electric potential, displacements, strain and electric field components may be expressed in the following matrices form:

$$
\left[\begin{array}{l}
u \\
\varphi
\end{array}\right]=\left([ \begin{array} { l l } 
{ A _ { u u } } & { A _ { u \varphi } } \\
{ 0 } & { A _ { \varphi \varphi } }
\end{array} ] \left[( \begin{array} { l } 
{ \mu _ { u } } \\
{ u _ { \varphi } }
\end{array} ] \left(, \quad\left[\begin{array}{l}
\varepsilon \\
E
\end{array}\right]=\left([ \begin{array} { l l } 
{ \mathcal { u u } _ { u } } & { L _ { u \varphi } } \\
{ 0 } & { L _ { \varphi \varphi } }
\end{array} ] \left[\left(\begin{array}{l}
\mu_{u} \\
\mu_{\varphi}
\end{array}\right](\right.\right.\right.\right.\right.
$$

where

$$
\begin{aligned}
& u_{u}=\left[\begin{array}{llllllll}
\mu_{0} & w_{0} & \theta & u_{1}^{1} & X^{+} & X^{-} & Z^{+} & Z^{-}
\end{array}\right]^{T}, \quad u_{\varphi}=\left[\begin{array}{lll}
\rho_{b}^{i} & \varphi_{c}^{i} & \varphi_{t}^{i}
\end{array}\right]^{T} \\
& u=\left[\begin{array}{ll}
\boldsymbol{u} & w
\end{array}\right]^{T}, \quad \varphi=\left[\begin{array}{ll}
\phi^{i}
\end{array}\right]^{T}, \quad \varepsilon=\left[\begin{array}{lll}
\varepsilon_{x x} & \varepsilon_{z z} & \gamma_{x z}
\end{array}\right]^{T}, \quad E=\left[\begin{array}{ll}
\mathscr{f}_{x} & E_{z}
\end{array}\right]^{T}
\end{aligned}
$$

and

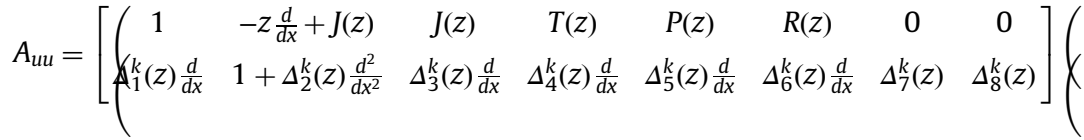

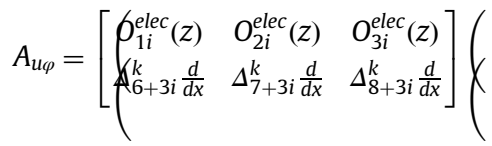

$$
\begin{aligned}
& A_{\varphi \varphi}=\left[\begin{array}{lll}
f_{1}^{i}(z) & L_{3}^{i}(z) & L_{2}^{i}(z)
\end{array}\right]( \\
& \left.L_{u u}=\left[\begin{array}{cccccccc}
\frac{d}{d x} & -z \frac{d^{2}}{d x^{2}}+J(z) \frac{d}{d x} & J(z) \frac{d}{d x} & T(z) \frac{d}{d x} & P(z) \frac{d}{d x} & R(z) \frac{d}{d x} & 0 & 0 \\
\left(\begin{array}{c}
k \\
1
\end{array}(z)_{, z} \frac{d}{d x}\right. & \Delta_{2}^{k}(z)_{, z} \frac{d^{2}}{d x^{2}} & \Delta_{3}^{k}(z)_{, z} \frac{d}{d x} & \Delta_{4}^{k}(z)_{, z} \frac{d}{d x} & \Delta_{5}^{k}(z)_{, z} \frac{d}{d x} & \Delta_{6}^{k}(z)_{, z} \frac{d}{d x} & \Delta_{7, z}^{k} & \Delta_{8, z}^{k} \\
0 & J(z)_{, z} \frac{d}{d x} & J(z)_{, z} & T(z)_{, z} & P(z)_{, z} & R(z)_{, z} & 0 & 0
\end{array}\right]\right)( \\
& L_{u \varphi}=\left[\left(\begin{array}{ccc}
O_{1 i}^{\text {elec }}(z) \frac{d}{d x} & O_{2 i}^{\text {elec }}(z) \frac{d}{d x} & O_{3 i}^{\text {elec }}(z) \frac{d}{d x} \\
\left(\Delta_{6+3 i}^{k}\right)_{, z} \frac{d}{d x} & \left(\Delta_{7+3 i}^{k}\right)_{, z} \frac{d}{d x} & \left(\Delta_{8+3 i}^{k}\right)_{, z} \frac{d}{d x} \\
\left(O_{1 i}^{\text {elec }}(z)_{, z}\right. & O_{2 i}^{\text {elec }}(z)_{, z} & O_{3 i}^{\text {elec }}(z)_{, z}
\end{array}\right]\right) \\
& \left.L_{\varphi \varphi}=-\left[\begin{array}{ccc}
f_{1}^{i}(z) \frac{d}{d x} & L_{3}^{i}(z) \frac{d}{d x} & L_{2}^{i}(z) \frac{d}{d x} \\
\left(\frac{d L_{1}^{i}(z)}{d z}\right. & \frac{d L_{3}^{i}(z)}{d z} & \frac{d L_{2}^{i}(z)}{d z}
\end{array}\right]\right\}
\end{aligned}
$$
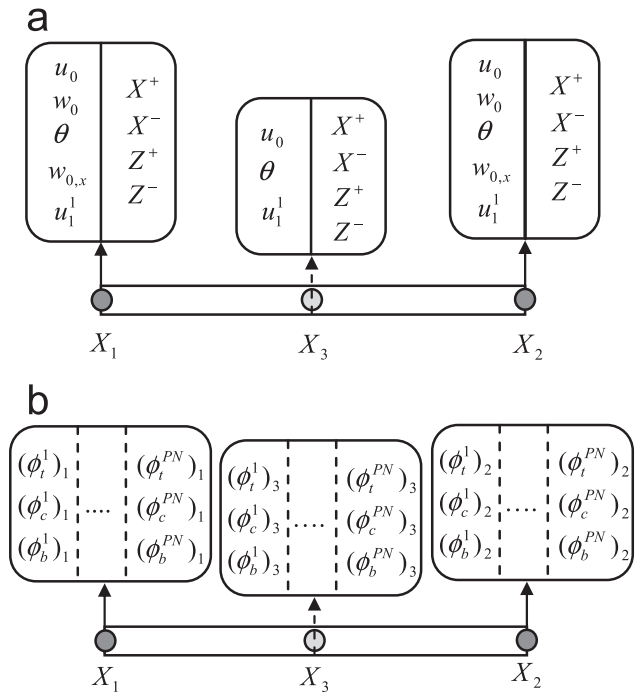

Fig. 3. The proposed piezoelectric beam element: (a) mechanical degrees of freedom and (b) electric potential degrees of freedom. 
The vector of displacement and electric potential components $\mathbf{u}_{u}$ and $u_{\varphi}$ may be expressed in terms of the mechanical and electrical nodal variables vectors $\mathbf{u}_{u}^{e}$ and $u_{\varphi}^{e}$ as follows:

$$
\left[\begin{array}{l}
\mathfrak{l}_{u} \\
\mathfrak{l}_{\varphi}
\end{array}\right]=\left[\begin{array}{cc}
\mathfrak{N}_{u u} & 0 \\
0 & N_{\varphi \varphi}
\end{array}\right]\left[\begin{array}{l}
\mathscr{\psi}_{u}^{e} \\
\mathfrak{l}_{\varphi}^{e}
\end{array}\right](
$$

where

$$
\begin{aligned}
& u_{u}^{e}=\left\{\begin{array}{lllllllllllllll}
\left(u_{0}\right)_{1} & \theta_{1} & \left(w_{0}\right)_{1} & \left(w_{0, x}\right)_{1} & \left(u_{1}^{1}\right)_{1} & \left(X^{-}\right)_{1} & \left(Z^{+}\right)_{1} & \left(Z^{-}\right)_{1} & \left(u_{0}\right)_{3} & \left(X^{+}\right)_{1} \theta_{3} & \left(u_{1}^{1}\right)_{3} & \left(X^{+}\right)_{3} & \left(X^{-}\right)_{3} & \left(Z^{+}\right)_{3} & \left(Z^{-}\right)_{3}
\end{array}\right. \\
& \begin{array}{llllll}
\left(u_{0}\right)_{2}\left(w_{0}\right)_{2} \theta_{2}\left(w_{0, x}\right)_{2} & \left(u_{1}^{1}\right)_{2} & \left(X^{+}\right)_{2} & \left(X^{-}\right)_{2} & \left(Z^{+}\right)_{2} & \left(Z^{-}\right)_{2}
\end{array}
\end{aligned}
$$

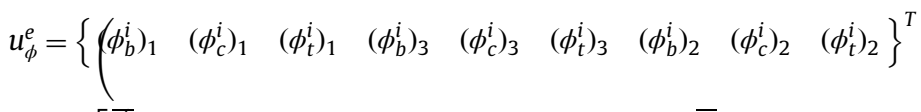

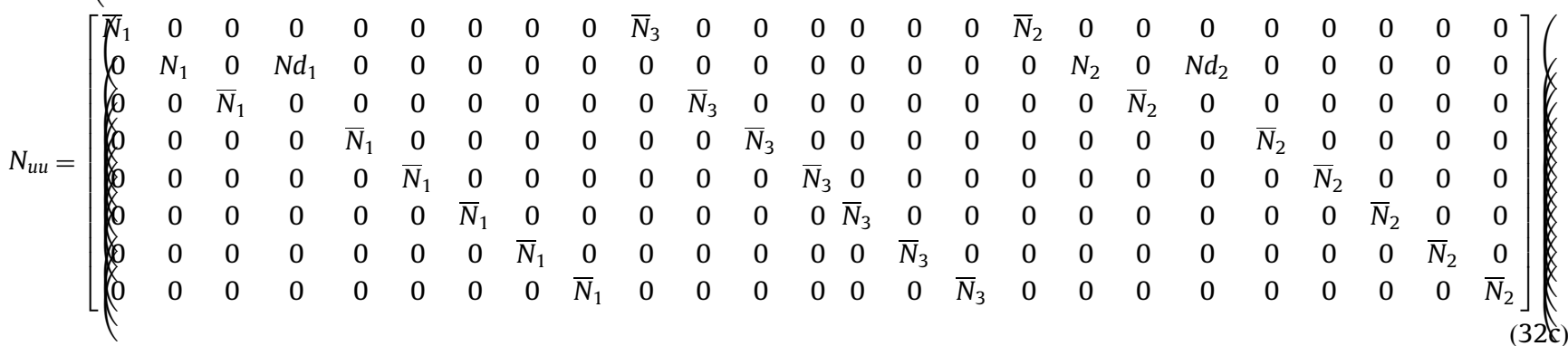

$$
\mathbf{N}_{\boldsymbol{\varphi \varphi}}=\left[\begin{array}{ccccccccc}
\bar{N}_{1} & 0 & 0 & \bar{N}_{3} & 0 & 0 & \bar{N}_{2} & 0 & 0 \\
0 & \bar{N}_{1} & 0 & 0 & \bar{N}_{3} & 0 & 0 & \bar{N}_{2} & 0 \\
0 & 0 & \bar{N}_{1} & 0 & 0 & \bar{N}_{3} & 0 & 0 & \bar{N}_{2}
\end{array}\right] \text { ( }
$$

in which $\bar{N}_{i}(i=1,2,3)$ are the Lagrangian quadratic shape functions defined as:

$$
\bar{N}_{1} \equiv \bar{N}_{1}(\xi)=-\xi(1-\xi) / 2, \quad \bar{N}_{2} \equiv \bar{N}_{2}(\xi)=\xi(1+\xi) / 2, \quad \bar{N}_{3} \equiv \bar{N}_{3}(\xi)=(1+\xi)(1-\xi)
$$

and the Hermitian shape functions are:

$$
\begin{aligned}
& N_{1}=N_{1}(\xi)=\frac{1}{4}(1-\xi)^{2}(2+\xi) \quad, \quad N_{2}=N_{2}(\xi)=\frac{1}{4}(2-\xi)(1+\xi)^{2} \\
& N d_{1}=N d_{1}(\xi)=\frac{l_{e}}{8}(1-\xi)^{2}(1+\xi) \quad, \quad N d_{2}=N d_{2}(\xi)=-\frac{l_{e}}{8}(1-\xi)(1+\xi)^{2}
\end{aligned}
$$

where $\xi$ is the natural coordinate and $l_{e}$ denotes the length of the element (see Fig. 4).

Using Eqs. (28) and (31), the displacements, electric potential, strain and electric field vectors may be expressed as follows:

$$
\begin{aligned}
& {\left[\begin{array}{l}
\mathbf{u} \\
\varphi
\end{array}\right]=\left[\begin{array}{cc}
\mathbf{A}_{\mathbf{u} u} & \mathbf{A}_{\mathbf{u} \varphi} \\
\mathbf{0} & \mathbf{A}_{\varphi \varphi}
\end{array}\right]\left[\begin{array}{l}
\boldsymbol{\mu}_{\mathbf{u}} \\
\mathbf{1}_{\varphi}
\end{array}\right]=\left[\begin{array}{cc}
\mathbf{A}_{\mathbf{u} u} & \mathbf{A}_{\mathbf{u} \varphi} \\
\mathbf{0} & \mathbf{A}_{\varphi \varphi}
\end{array}\right]\left[\begin{array}{cc}
\mathbf{N}_{\mathbf{u u}} & \mathbf{0} \\
\mathbf{0} & \mathbf{N}_{\varphi \varphi \varphi}
\end{array}\right]\left[\begin{array}{l}
\mathbf{\mu}_{u}^{e} \\
\mathbf{u}_{\varphi}^{\mathbf{e}}
\end{array}\right]\left(=\mathcal{N} \mathbf{u}^{e}\right.}
\end{aligned}
$$

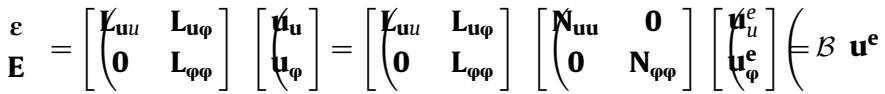

In Eq. (35a), $\mathcal{N}$ denotes the displacements and the electric potential interpolation matrix. Strains and electric fields interpolation matrix is represented with $\mathcal{B}$.

In the present study, the principle of virtual work is employed to extract the governing equations of the piezoelectric beam element. According to this principle, for a piezoelectric medium of volume $\Omega$ and regular boundary surface $\Gamma$, one may write [33]:

$$
\delta \Pi=\delta U+\delta W=-\int_{\Omega} \delta \varepsilon^{T} \sigma d \Omega+\iint_{f} \delta u^{T} F_{S} d \Gamma+\int_{\Omega} \delta u^{T} F_{V} d \Omega-\iint_{\ell} \rho \delta u^{T} \ddot{u} d \Omega+\int_{\Omega} \delta E^{T} D d \Omega-\int_{\Gamma} \bar{Q} \delta \varphi d \Gamma-\iint_{\ell} \bar{q} \delta \varphi d \Omega=0
$$

where $\mathbf{F}_{S}, \mathbf{F}_{\mathbf{V}}, \bar{q}, \bar{Q}$ and $\rho$ are surface force vector, mechanical body force vector, electrical body charge, surface charge and mass density, respectively. $\delta \mathbf{u}$ and $\delta \varphi$ are admissible virtual displacement and potential. Substituting Eqs. (3) and (35) into Eq. (36), and assembling the

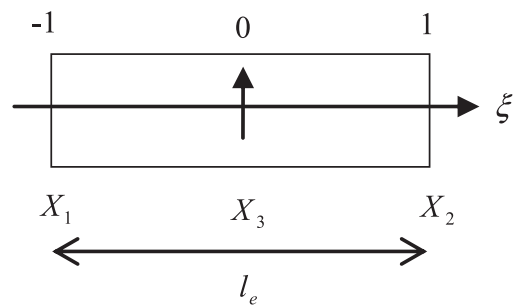

Fig. 4. The proposed element and its longitudinal local coordinate. 
element equations, yields the following general static equation:

$$
\mathbf{K} \mathbf{q}=\mathbf{F}
$$

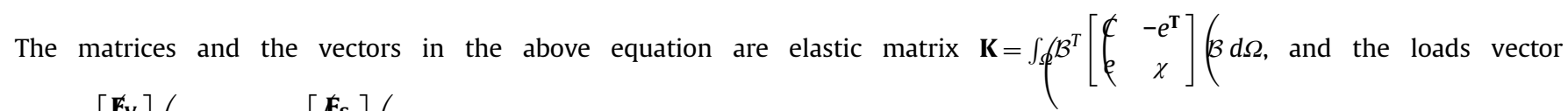
$\mathbf{F}=\int_{\phi} \mathcal{N}^{T}\left[\begin{array}{l}\mathbf{F}_{\mathbf{v}} \\ f \bar{q}\end{array}\right]\left(d \Omega+\int_{l} \mathcal{N}^{T}\left[\begin{array}{c}F_{\mathbf{S}} \\ f \bar{Q}\end{array}\right](d \Gamma\right.$

Table 1

The material properties of the graphite-epoxy and PZT-5A shear actuator or sensor.

\begin{tabular}{|c|c|c|}
\hline Property & Graphite-epoxy & PZT-5A \\
\hline$C_{11}(\mathrm{GPa})$ & 183.443 & 86.856 \\
\hline$C_{22}(\mathrm{GPa})$ & 11.662 & 99.201 \\
\hline$C_{33}(\mathrm{GPa})$ & 11.662 & 99.201 \\
\hline$C_{12}(\mathrm{GPa})$ & 4.363 & 50.778 \\
\hline$C_{13}(\mathrm{GPa})$ & 4.363 & 50.778 \\
\hline$C_{23}(\mathrm{GPa})$ & 3.918 & 54.016 \\
\hline $\mathrm{C}_{44}(\mathrm{GPa})$ & 2.870 & 22.593 \\
\hline$C_{55}(\mathrm{GPa})$ & 7.170 & 21.100 \\
\hline$C_{66}(\mathrm{GPa})$ & 7.170 & 21.100 \\
\hline$C_{16}(\mathrm{GPa})$ & 0 & 0 \\
\hline$C_{26}(\mathrm{GPa})$ & 0 & 0 \\
\hline$C_{36}(\mathrm{GPa})$ & 0 & 0 \\
\hline $\mathrm{C}_{45}(\mathrm{GPa})$ & 0 & 0 \\
\hline$e_{11}\left(\mathrm{~cm}^{-2}\right)$ & 0 & 15.118 \\
\hline$e_{12}\left(\mathrm{~cm}^{-2}\right)$ & 0 & -7.209 \\
\hline$e_{13}\left(\mathrm{~cm}^{-2}\right)$ & 0 & -7.209 \\
\hline$e_{16}\left(\mathrm{~cm}^{-2}\right)$ & 0 & 0 \\
\hline$e_{21}\left(\mathrm{~cm}^{-2}\right)$ & 0 & 0 \\
\hline$e_{22}\left(\mathrm{~cm}^{-2}\right)$ & 0 & 0 \\
\hline$e_{23}\left(\mathrm{~cm}^{-2}\right)$ & 0 & 0 \\
\hline$e_{26}\left(\mathrm{~cm}^{-2}\right)$ & 0 & 12.322 \\
\hline$e_{34}\left(\mathrm{~cm}^{-2}\right)$ & 0 & 0 \\
\hline$e_{35}\left(\mathrm{~cm}^{-2}\right)$ & 0 & 12.322 \\
\hline$\chi_{11}\left(10^{-10} \mathrm{Fm}^{-1}\right)$ & 153.0 & 150.0 \\
\hline$\chi_{22}\left(10^{-10} \mathrm{Fm}^{-1}\right)$ & 153.0 & 153.0 \\
\hline$\chi_{33}\left(10^{-10} \mathrm{Fm}^{-1}\right)$ & 153.0 & 153.0 \\
\hline$\chi_{12}\left(10^{-10} \mathrm{Fm}^{-1}\right)$ & 0 & 0 \\
\hline
\end{tabular}

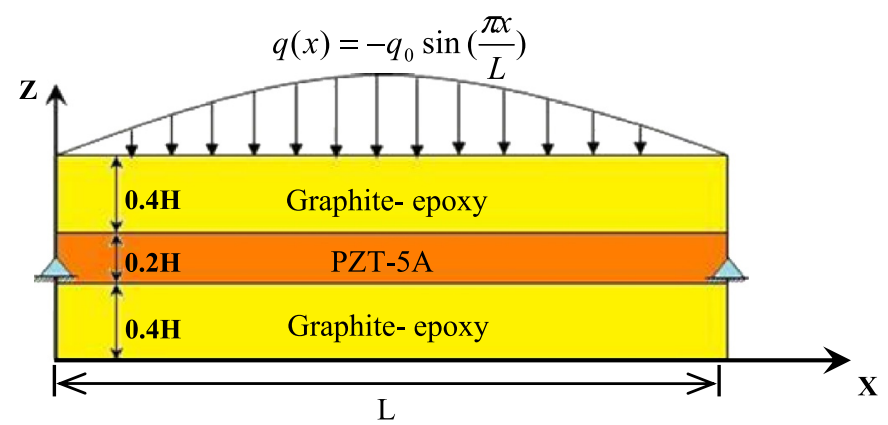

Fig. 5. Characteristics of the geometry, boundary conditions and loading of the sandwich beam including shear piezoelectric core.

Table 2

Results of the mesh convergence study for the sandwich beam with $S=10$.

\begin{tabular}{|c|c|c|c|c|c|c|}
\hline & \multicolumn{5}{|c|}{ No. element } & \multirow[t]{2}{*}{ Exact } \\
\hline & 3 & 5 & 9 & 12 & 15 & \\
\hline$\tilde{w}(0.5 L, 0)$ & 16.919 & 16.960 & 16.966 & 16.966 & 16.966 & 16.938 \\
\hline$\tilde{u}(0,0.5 H)$ & -2.3097 & -2.3094 & -2.3093 & -2.3093 & -2.3093 & -2.2864 \\
\hline$\tilde{\sigma}_{X X}(0.5 L, 0.5 H)$ & 60.079 & 61.866 & 62.571 & 63.242 & 62.773 & 62.267 \\
\hline$\tilde{\sigma}_{\mathrm{Xz}}(0,0)$ & 3.9972 & 4.4586 & 4.6456 & 4.6825 & 4.6996 & 4.6845 \\
\hline$\tilde{\sigma}_{z z}(0.5 L, 0)$ & 0.5004 & 0.5004 & 0.5004 & 0.5004 & 0.5004 & 0.5003 \\
\hline$E_{\mathrm{z}}(0,0)$ & -0.1035 & -0.1029 & -0.1028 & -0.1028 & -0.1028 & -0.1028 \\
\hline
\end{tabular}


Eq. (37) can be partitioned and re-arranged as:

$$
\left[\begin{array}{ll}
\mathbf{K}_{\mathbf{q q}} & \mathbf{K}_{\mathbf{q} \varphi} \\
\mathbf{K}_{\varphi q} & \mathbf{K}_{\varphi \varphi}
\end{array}\right]\left[\begin{array}{l}
\mathbf{q}_{\mathbf{u}} \\
\mathbf{q}_{\varphi}
\end{array}\right]\left(=\left[\begin{array}{l}
\mathbf{F}_{\mathbf{u}} \\
\mathbf{F}_{\varphi}
\end{array}\right](\right.
$$

where $\mathbf{q}_{\mathbf{u}}$ and $\mathbf{q}_{\boldsymbol{\varphi}}$ are the vectors of mechanical degrees of freedom and electrical degrees of freedom, respectively.

\section{Numerical results and discussions}

To assess the performance and the validity of the developed coupled theory, some examples of sandwich beams including shear piezoelectric layer (actuator/sensor) in the different boundary condition have been analyzed using the presented finite element model. The results of the present finite element are compared with the 3D exact piezoelasticity solution [42] and the 3D finite element (ABAQUS) results. The present numerical results are obtained from a MATLAB program whose algorithm is based on the theoretical formulation described in the previous section. The program allows any element at any layer to be made of different materials (piezoelectric or nonpiezoelectric).

\subsection{Example 1}

A simply supported piezoelectric sandwich beam with length to thickness ratio $S(L / h)=4$ (thick beam), $S=10$ (moderately thick beam) and $S=40$ (thin beam) is analyzed using the present coupled theory. As shown in Fig. 4, we consider a sandwich beam with the top and the bottom layers made of graphite-epoxy and the central layer made of PZT-5A shear actuator or sensor. The ratio of the piezoelectric thickness layer to the laminate thickness $h$ is assumed to be 0.2 and the non-piezoelectric layers are assumed to have equal thickness. The material properties of the graphite-epoxy and PZT-5A, shear actuator or sensor, shear actuator or sensor are given in Table 1.

\subsubsection{Sensor case}

A sinusoidal pressure $q(x)=-q_{0} \sin (\pi x / L)$ has been applied on the top surface of the simply supported beam (Fig. 5). Results of the mesh convergence study are shown in Table 2 for $S=10$.The obtained numerical results are normalized as follows:

$$
\tilde{u}_{i}=u_{i} C_{0} / L q_{0} \quad \tilde{\sigma}_{i j}=\sigma_{i j} / q_{0}
$$

Herein, $C_{0}=21.1 \mathrm{GPa}$ is the representative value of the elastic modulus for PZT-5A.

Table 2 shows that the convergence rate of the proposed finite element model is very high. A mesh with 3 elements gives excellent results in the prediction of deflection of the beam. Only 5 elements are adequate to predict the induced sensory electric field in the piezoelectric layer and stress components. However, for the analysis of the problem, the beam was mathematically divided into 15 beam elements of equal lengths and three layers.

The normalized numerical results for deflection, in-plane displacement, transverse shear and normal stresses, the induced electric field at the sensory layer and in-plane stress are given in Table 3, for three values of length to thickness ratios $S=4, S=10$ and $S=40$. The variation of the normalized stress and the displacement components $\left(\tilde{\sigma}_{x z}, \tilde{\sigma}_{x x}, \tilde{u}, \tilde{w}\right)$ through the thickness $(S=4$ and $S=40)$ are shown in Fig. 6 and Fig. 7. The distribution of the induced electric field $\left(E_{z}\right)$ across the sensory layer is also shown in these figures. Through-thethickness variation of the normalized transverse normal stress is depicted in Fig. 8. Note that the transverse normal stresses have been calculated using two different methods: (i) employing the constitutive equations; (ii) integrating the elasticity equations in terms of the stress components, across the thickness of the beam. Results of these two approaches are shown in Fig. 8 by (C) and (E), respectively.

Table 3

\begin{tabular}{|c|c|c|c|c|}
\hline & $\mathrm{S}$ & Present & Error (\%) & Exact \\
\hline \multirow[t]{3}{*}{$\tilde{w}(0.5 L, 0)$} & 4 & 1.9332 & 0.23 & 1.9287 \\
\hline & 10 & 16.9601 & 0.13 & 16.9378 \\
\hline & 40 & 930.278 & 0.01 & 930.191 \\
\hline \multirow[t]{3}{*}{$\tilde{u}(0,0.5 H)$} & 4 & -0.4142 & 3.8 & -0.3990 \\
\hline & 10 & -2.3093 & 1 & -2.2864 \\
\hline & 40 & -36.1758 & 0 & -36.1758 \\
\hline \multirow{3}{*}{$\tilde{\sigma}_{x x}(0.5 L, 0.5 H)$} & 4 & 11.4028 & 2.02 & 11.1770 \\
\hline & 10 & 62.5706 & 0.48 & 62.2667 \\
\hline & 40 & 974.686 & 0.43 & 978.984 \\
\hline \multirow[t]{3}{*}{$\tilde{\sigma}_{\mathrm{Xz}}(0,0)$} & 4 & 1.8957 & 0.02 & 1.8953 \\
\hline & 10 & 4.6825 & 0.04 & 4.6845 \\
\hline & 40 & 18.5128 & 0.96 & 18.6929 \\
\hline \multirow[t]{3}{*}{$\tilde{\sigma}_{z z}(0.5 L, 0)$} & 4 & 0.5027 & 0.47 & 0.5003 \\
\hline & 10 & 0.5004 & 0.01 & 0.5003 \\
\hline & 40 & 0.50002 & 0 & 0.50002 \\
\hline \multirow[t]{3}{*}{$E_{\mathrm{Z}}(0,0)$} & 4 & -0.0409 & 0.96 & -0.0413 \\
\hline & 10 & -0.1028 & 0 & -0.1028 \\
\hline & 40 & -0.4114 & 0.14 & -0.4108 \\
\hline
\end{tabular}

Numerical results of the sandwich beam is subjected to the mechanical load. 
For deflection, the present model predicts the results with an error less than $0.23 \%$ for any length to thickness ratio. The model predicts the in-plane displacement and in-plane stress at the top face of the thick laminated beam with the maximum error of 3.8\% and 2.02\%, respectively. The error approaches zero for thin beams $(S=40)$. The transverse shear and normal stress distributions obtained from the present model are in excellent agreement with the exact solution for both thin and thick laminated beams. The coupled proposed theory is
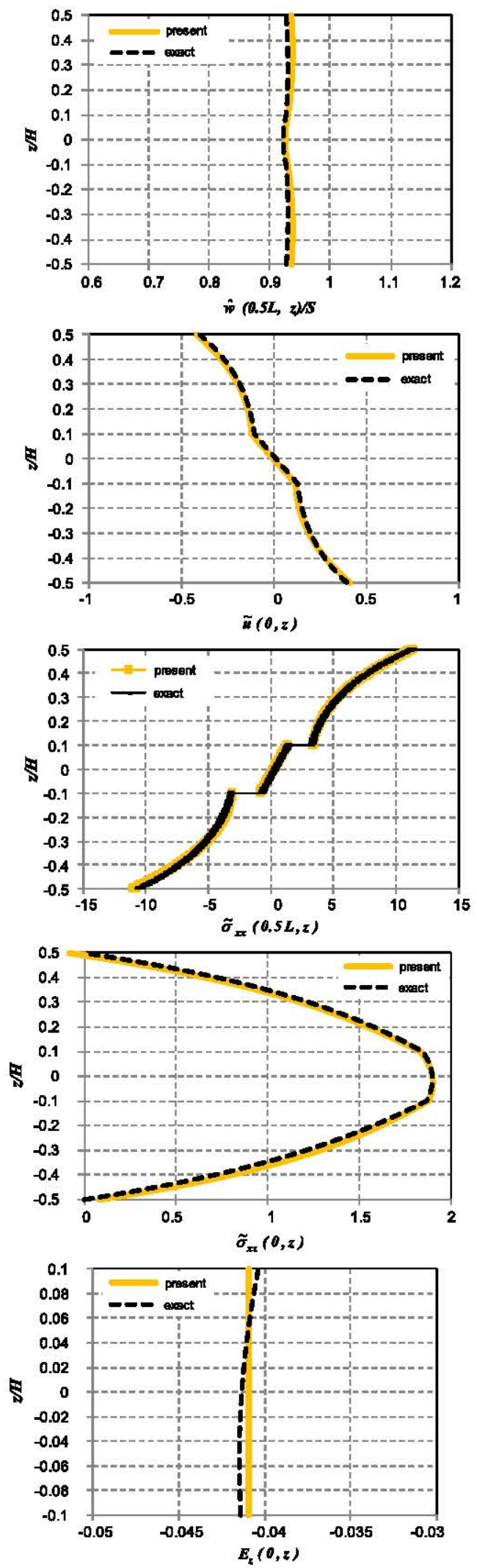

Fig. 6. Through-thickness distribution of $\tilde{w}, \tilde{u}, \tilde{\sigma}_{x x}, \tilde{\sigma}_{x z}$ and $E_{z}$ for the simply supported sandwich beam $(S=4)$. 

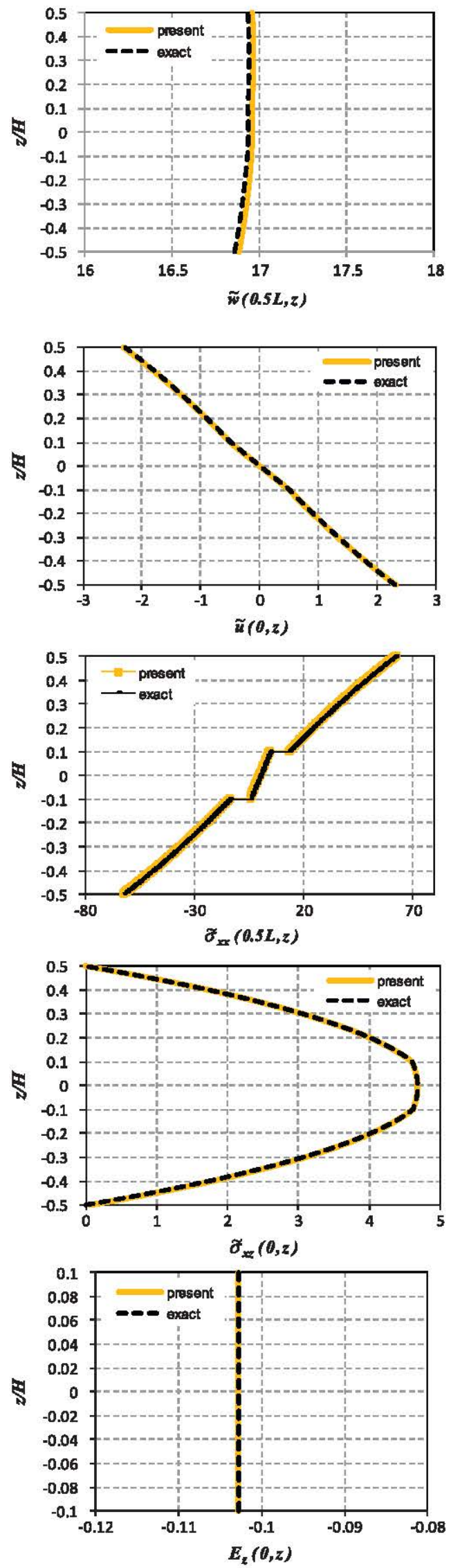

Fig. 7. Through-thickness distribution of $\tilde{w}, \tilde{u}, \tilde{\sigma}_{x x}, \tilde{\sigma}_{x z}$ and $E_{z}$ for the simply supported sandwich beam $(S=40)$. 
also capable of predicting the induced sensory electric field with very good accuracy for thin to thick laminated beams. The error in the predicted sensory electric field is only $0.96 \%$ for thick beams. The error approaches zero for thinner beams.

It can be seen that the present theory is able to accurately predict the transverse normal stress from the constitutive equations. Variations of the normalized deflection calculated at the middle of the sensory sandwich beam versus the aspect ratio are shown in Fig. 9. Comparison of the present results with the results of the exact piezoelectricity solution reveals that the present beam element is free of shear locking. These results demonstrate that the developed finite element model performs well in the prediction of the sensory behavior of thick and thin sandwich beams.
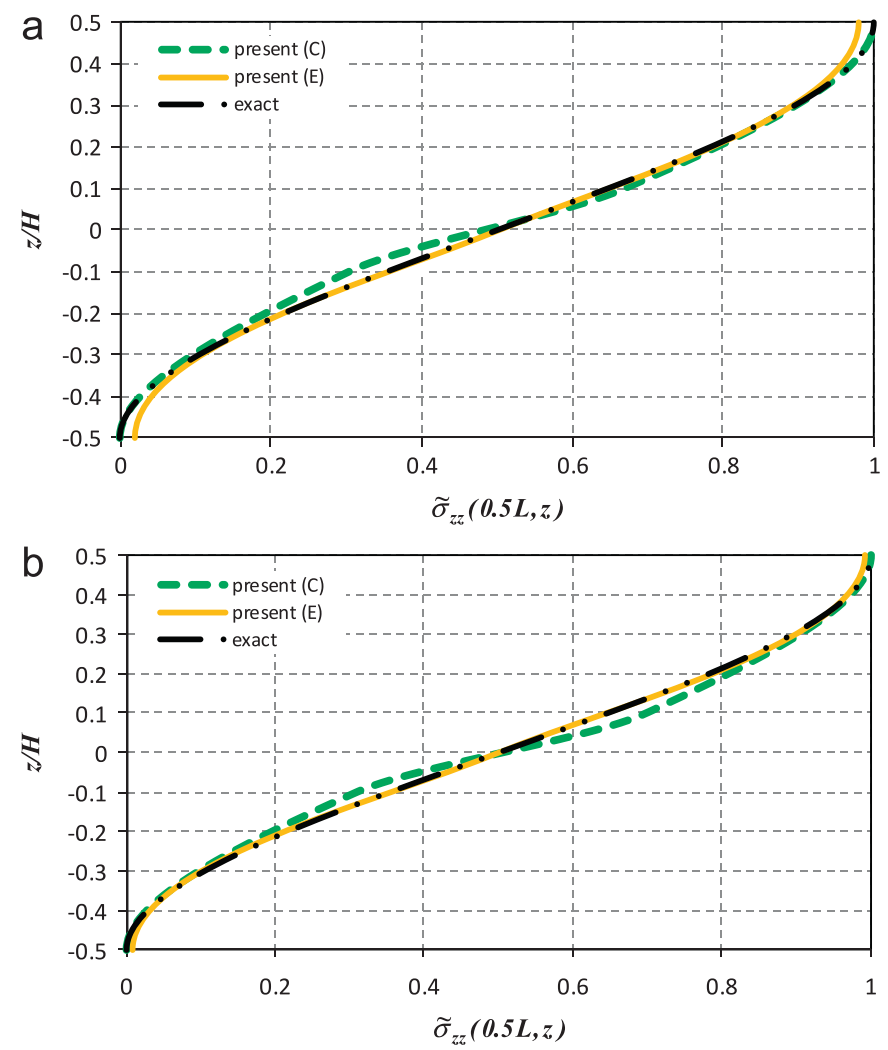

Fig. 8. Through-thickness distribution of normalized transverse normal stress (a) $S=4$ and (b) $S=10$.

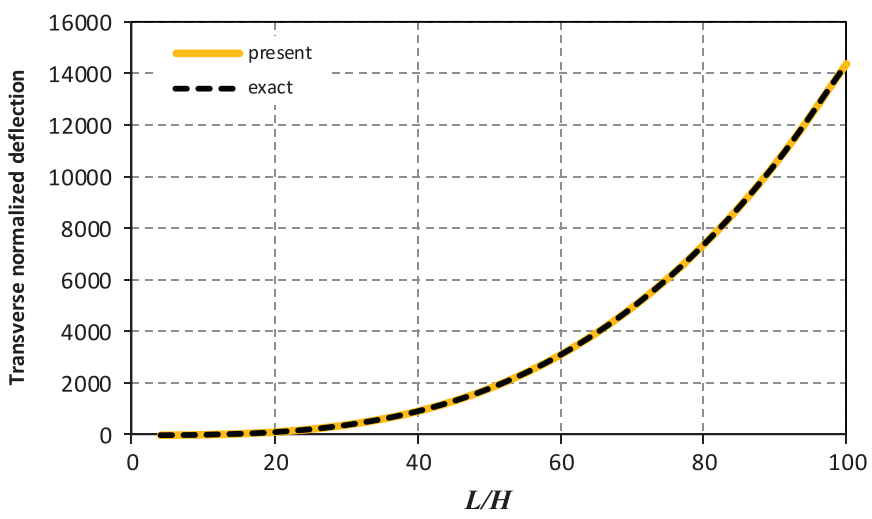

Fig. 9. Variations of $\tilde{w}(0.5 L, 0)$ versus the aspect ratio for the sensory sandwich beam.

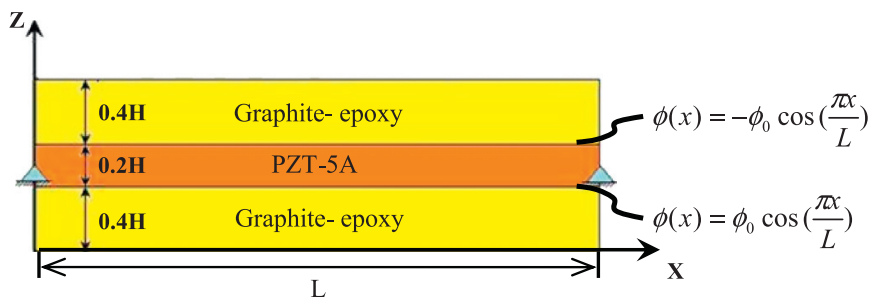

Fig. 10. Characteristics of the geometry of the sandwich beam including shear piezoelectric actuator and the actuating potential. 


\subsubsection{Actuator case}

The actuating potential $\phi(x)=-\phi_{0} \cos (\pi x / L)$ and $\phi(x)=\phi_{0} \cos (\pi x / L)$ has been applied on the top and bottom surfaces of the piezoelectric layer, respectively (Fig. 10).Table 4 shows the results of the mesh convergence test for $S=4$. Similar to the sensor case, the convergence rate of the present finite element is very high. The obtained numerical results are normalized as follows:

$$
\hat{u}_{i}=u_{i} C_{0} / e_{0} \phi_{0} \quad \hat{\sigma}_{i j}=\sigma_{i j} L / e_{0} \phi \quad \hat{D}_{i}=D_{i} / \chi_{0} \phi_{0}
$$

where $C_{0}=21.1 \mathrm{GPa}, e_{0}=12.322 \mathrm{Cm}^{-2}$ and $\chi_{0}=153 \times 10^{-10} \mathrm{Fm}^{-1}$ are representative values of the elastic and piezoelectric modulus and the electric permittivity, respectively for PZT-5A $\left(\phi_{0}=1\right.$.)

The normalized numerical results for the transverse electric displacement, stress and displacement components are compared in Table 5 for length to thickness ratios $S=4, S=10$ and $S=40$.

The model predicts the in-plane displacement, deflection and in-plane stresses of thin to thick sandwich beams with an error less than 3.05\%. For transverse electric displacement, the present theory predicts the results with a maximum error of $0.38 \%$, for any length to thickness ratio. Similar to the sensor model, the transverse shear stress distributions deduced from the present model are in excellent agreement with the exact solution. The error in case of thick beams, $S=4$ is less than $2.55 \%$. In cases with higher values of $S$ (thinner widths), the accuracy is even more pronounced. Through-the-thickness distributions of $\hat{w}, \hat{u}, \hat{\sigma}_{x x}, \hat{\sigma}_{z z}, \hat{\sigma}_{x z}$ and $\hat{D}_{z}$ for thick and thin beams are shown in Figs. 11 and 12.

Considering the various values for the aspect ratio, the normalized deflection of the active sandwich beam is shown in Fig. 13 along with the exact piezoelectricity solutions. It can be inferred from this figure that the present finite element does not suffer from shear lucking. Furthermore, these results demonstrate that the proposed model is efficient in predicting the actuator behavior of thick and thin smart sandwich beams.

Table 4

Results of the mesh convergence study for the smart sandwich beam with $S=4$.

\begin{tabular}{|c|c|c|c|c|c|c|}
\hline & \multicolumn{5}{|c|}{ No. element } & exact \\
\hline$\hat{w}(0.5 L, 0)$ & 3.6941 & 3.7031 & 3.7044 & 3.7047 & 3.7047 & 3.6911 \\
\hline$\hat{\sigma}_{x x}(0.5 L, 0.5 H)$ & 11.0616 & 11.3937 & 11.5244 & 11.6488 & 11.5619 & 11.2403 \\
\hline$\hat{\sigma}_{x z}(0,0)$ & -0.8902 & -0.9928 & -1.0347 & -1.0430 & -1.0468 & -1.0436 \\
\hline $\max \left(\hat{\sigma}_{z z}(0.5 L, z)\right)$ & 0.0994 & 0.1024 & 0.1036 & 0.1048 & 0.1039 & 0.1098 \\
\hline
\end{tabular}

Table 5

Numerical results of the sandwich beam is subjected to the actuating potential.

\begin{tabular}{|c|c|c|c|c|}
\hline & $\mathrm{S}$ & Present & Error (\%) & Exact \\
\hline \multirow[t]{3}{*}{$\hat{w}(0.5 L, 0)$} & 4 & 3.7031 & 0.32 & 3.6911 \\
\hline & 10 & 9.2638 & 0.14 & 9.2505 \\
\hline & 40 & 37.070 & 0.11 & 37.030 \\
\hline \multirow[t]{3}{*}{$\hat{w}(0.5 L, 0.5 H)$} & 4 & 3.7440 & 0.9 & 3.7103 \\
\hline & 10 & 9.2767 & 0.14 & 9.2631 \\
\hline & 40 & 37.073 & 0.11 & 37.033 \\
\hline \multirow[t]{3}{*}{$\hat{u}(0,0.5 H)$} & 4 & -0.4279 & 3.03 & -0.4153 \\
\hline & 10 & -0.4448 & 0.67 & -0.4471 \\
\hline & 40 & -0.4481 & 1.34 & -0.4541 \\
\hline \multirow[t]{3}{*}{$\hat{\sigma}_{\mathrm{xx}}(0.5 L, 0.5 H)$} & 4 & 11.3937 & 1.36 & 11.2403 \\
\hline & 10 & 12.1106 & 0.05 & 12.1044 \\
\hline & 40 & 12.1998 & 0.76 & 12.2940 \\
\hline \multirow[t]{3}{*}{$\hat{\sigma}_{\mathrm{Xz}}(0,0)$} & 4 & -1.0430 & 0.05 & -1.0436 \\
\hline & 10 & -0.4434 & 2.12 & -0.4530 \\
\hline & 40 & -0.1121 & 2.52 & -0.1150 \\
\hline \multirow[t]{3}{*}{$\hat{D}_{z}(0,0.1 H)$} & 4 & 14.6361 & 0.15 & 14.6135 \\
\hline & 10 & 14.6435 & 0.29 & 14.6872 \\
\hline & 40 & 14.6450 & 0.38 & 14.7020 \\
\hline
\end{tabular}




\subsection{Example 2}

In this example, a cantilever smart sandwich beam with length $L=10 \mathrm{~cm}$, cross section width $b=1 \mathrm{~cm}$ and height $h=1 \mathrm{~cm}$ is considered. Thickness of the piezoelectric layer is $0.2 \mathrm{~h}$ and thickness of the face sheets is assumed to be $0.4 \mathrm{~h}$. The face sheets are made of graphite-epoxy and the central layer made of PZT-5A shear actuator or sensor. Material properties of the smart sandwich beam of the present example are the same as those of the previous example. Since no exact 3D piezoelasticity solution is available, for the considered example, a coupled 3D finite element analysis was performed in ABAQUS with a very refined mesh, using the 20-node piezoelectric solid element (C3D20RE). It should be noted that, the displacements and stresses are normalized as

$$
\hat{u}_{i}=u_{i} C_{0} / e_{0} \phi_{0} \quad \hat{\sigma}_{i j}=\sigma_{i j} L / e_{0} \phi_{0}
$$

for the applied electric load and

$$
\tilde{u}_{i}=u_{i} C_{0} / L q_{0} \quad \tilde{\sigma}_{i j}=\sigma_{i j} / q_{0}
$$

for the applied mechanical load. Here $C_{0}=21.1 \mathrm{GPa}$ and $e_{0}=12.322 \mathrm{Cm}^{-2}$ are representative values of the elastic and piezoelectric modulus, respectively for PZT-5A.

\subsubsection{Sensor case}

The sensory sandwich beam is subjected to distributed sinusoidal pressureq $(x)=-\sin (\pi x / L)$ on its top surface (Fig. 14). In order to analyze the problem, the beam was mathematically divided into 15 beam elements of equal lengths. In Fig. 15, through-the-thickness distributions of the stress and displacement components and the induced sensory electric field are shown. It may be observed that the depicted in-plane displacement component based on the present formulation is in excellent agreement with the coupled 3D finite element results. The error in present finite element results is $2.8 \%$ for the in-plane stress, less than $6 \%$ for the sensory electric field and
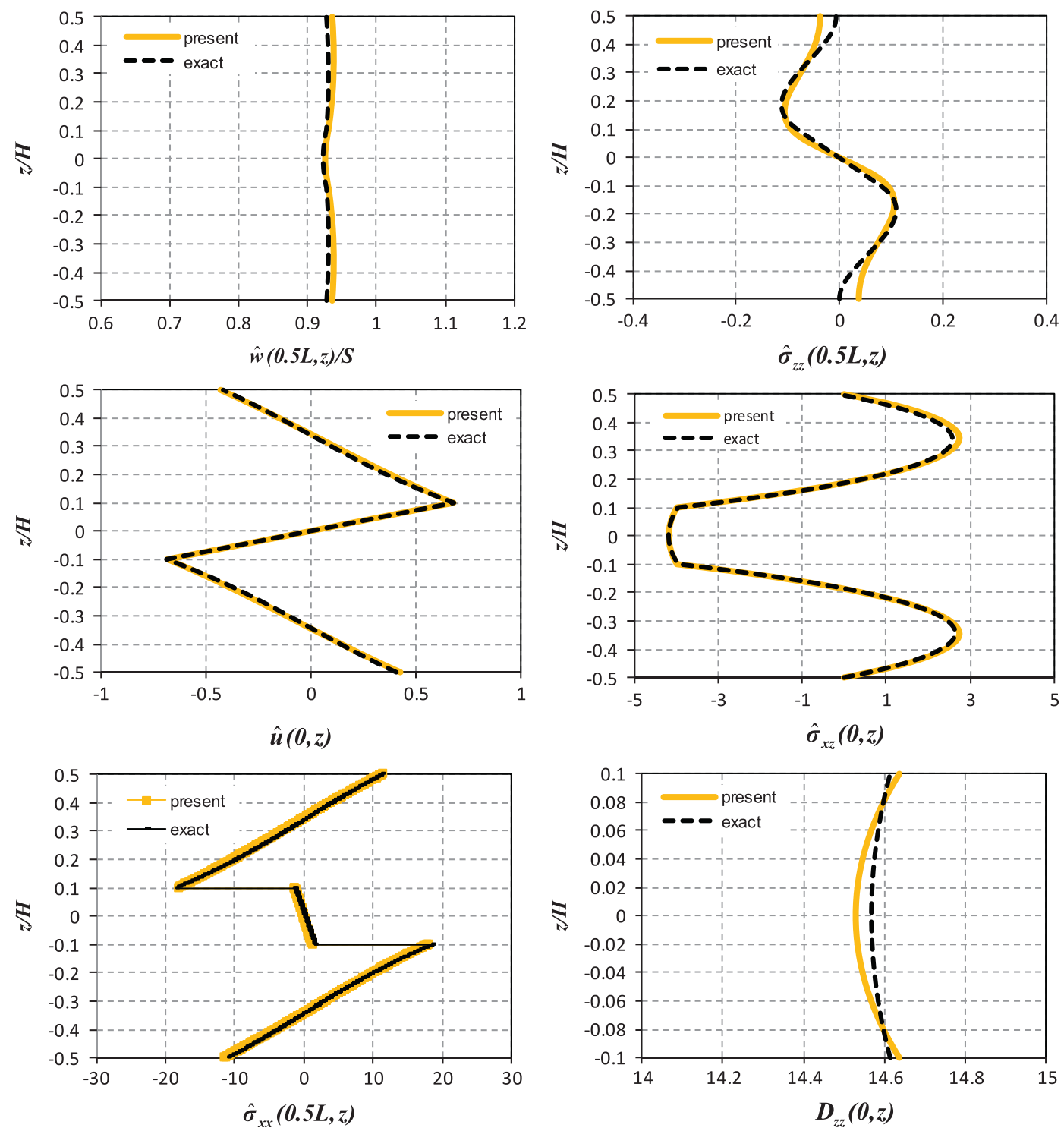

Fig. 11. Through-thickness distribution of the stress and displacement components and the transverse electric displacement for the active sandwich beam $(S=4)$. 

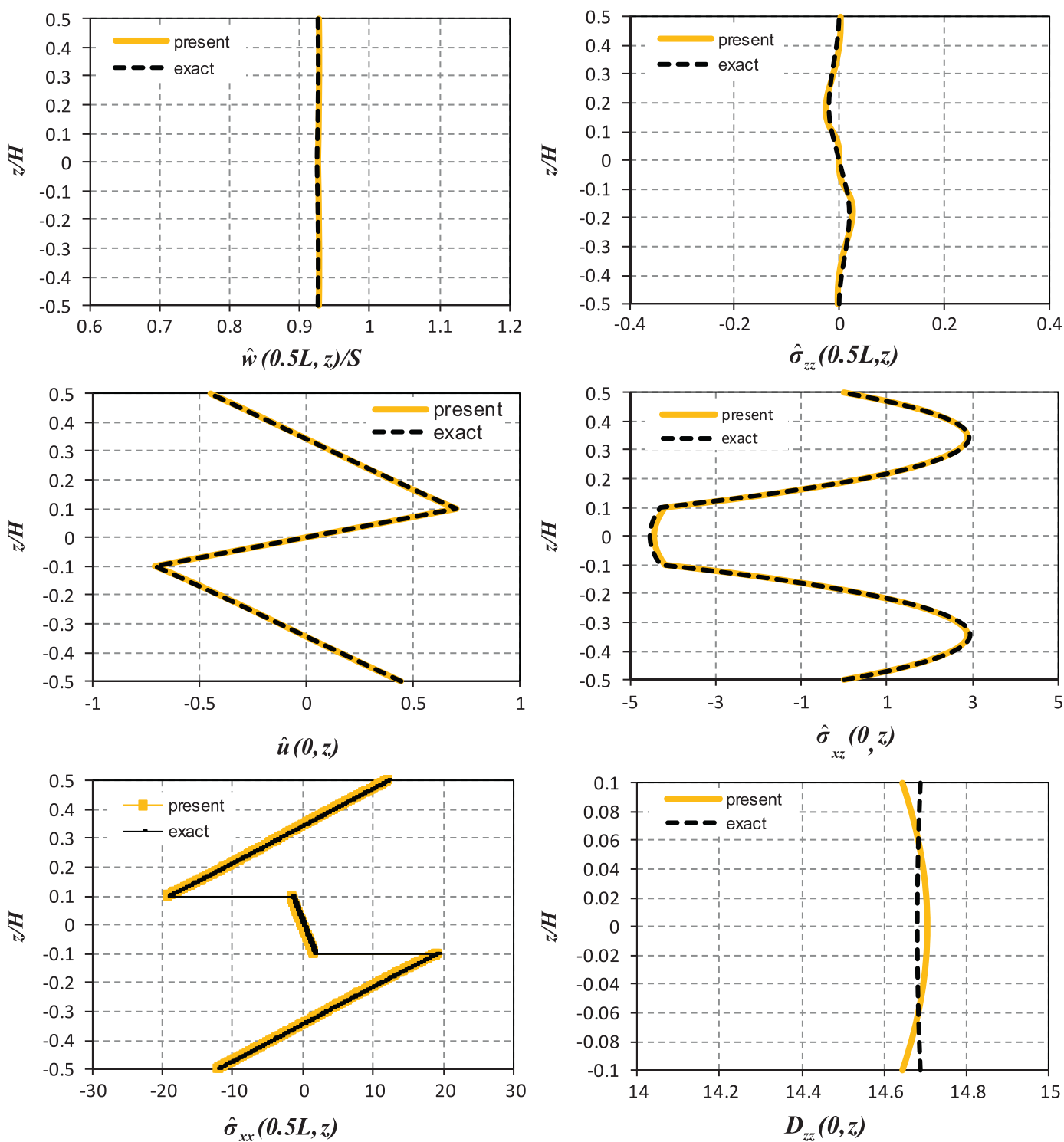

Fig. 12. Through-thickness distribution of the stress and displacement components and the transverse electric displacement for the active sandwich beam ( $S=40$ ).

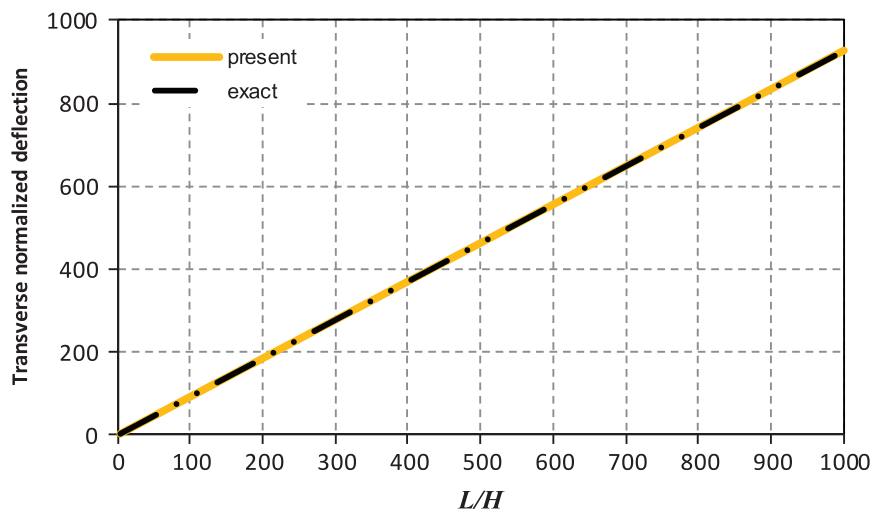

Fig. 13. The normalized transverse deflection versus the aspect ratio for the active sandwich beam.

$0.18 \%$ for the transverse displacement. The proposed finite element model predicts the transverse shear stresses of the sensory sandwich beam with an error less than $1.5 \%$. Moreover, the transverse normal stresses predicted from the constitutive equations are also in good agreement with those extracted from the coupled 3D finite element analysis. These results confirm the accuracy of the proposed formulation in the prediction of the sensory behavior of sandwich beams. 


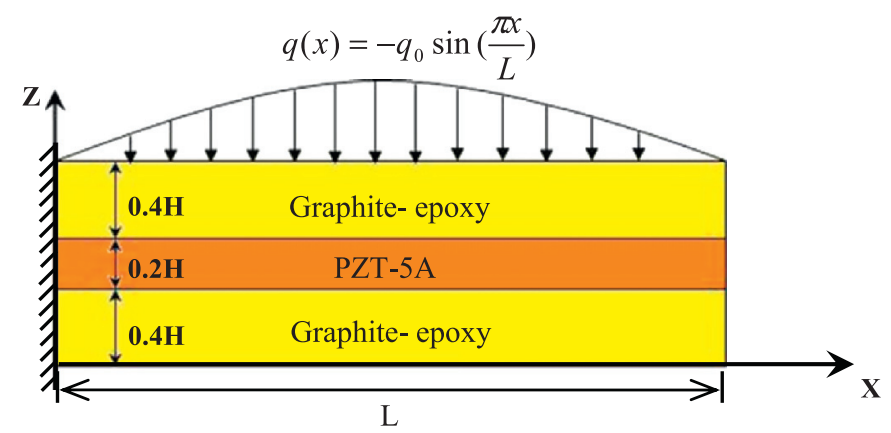

Fig. 14. Characteristics of the geometry, boundary conditions and loading of the cantilever sandwich beam including shear piezoelectric core.
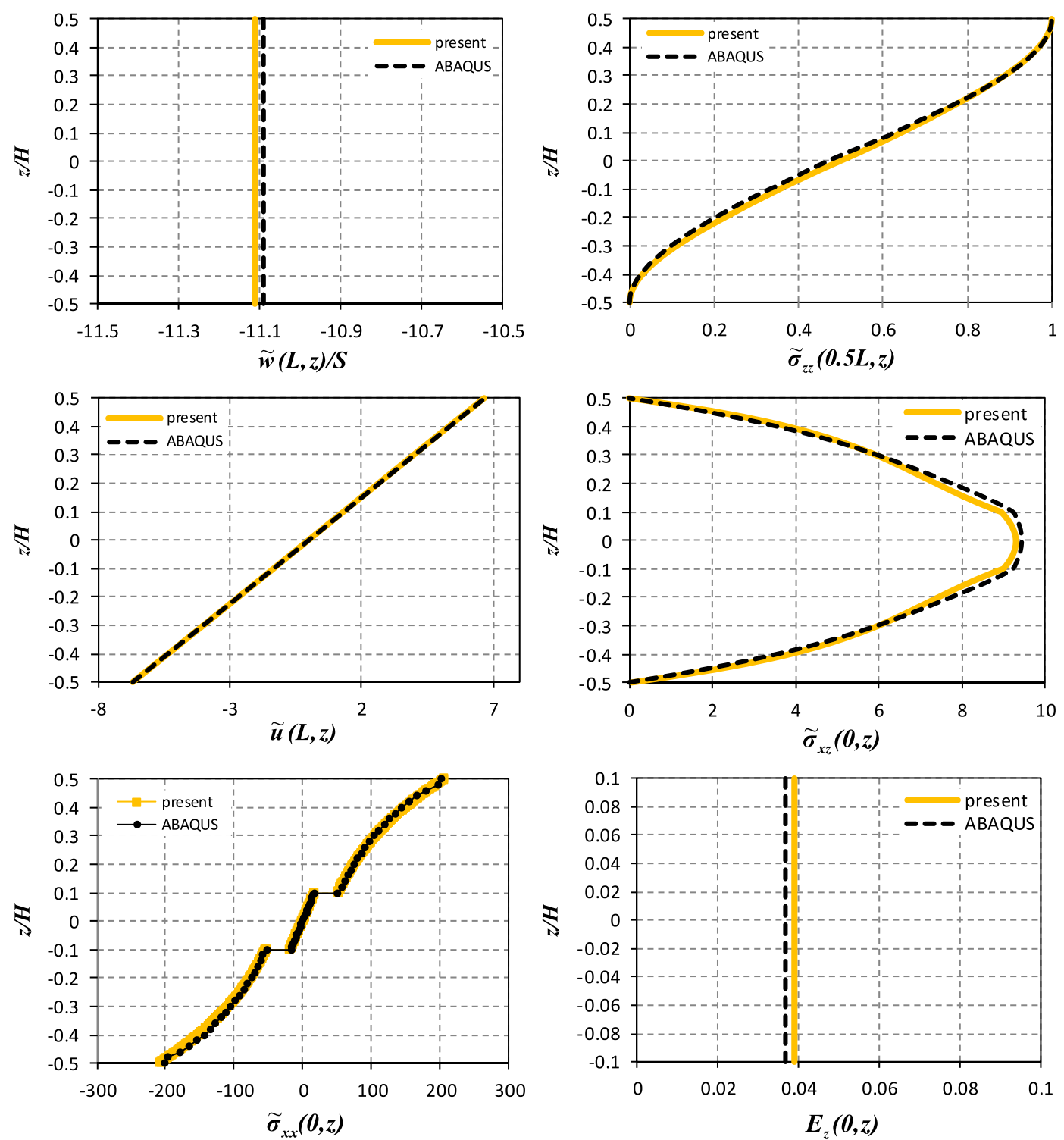

Fig. 15. Variations of the stress and displacement components and the induced transverse electric field for the cantilever sandwich beam.

\subsubsection{Actuator case}

In this case, the cantilever sandwich shear piezoelectric sandwich beam is subjected to the actuating potential $\phi(x)=-\phi_{0} \cos (\pi x / L)$ and $\phi(x)=\phi_{0} \cos (\pi x / L)$ on its top and bottom surfaces, respectively (Fig. 16). The results corresponding to this case study are plotted in Fig. 17.

As shown in Fig. 17, the prediction of the deflection by the present finite element model agrees well with the results of ABAQUS due to the inclusion of the effects of both the transverse flexibility and the electrical transverse normal strains. The model predicts the maximum deflection of the active sandwich beam with an error of less than $0.5 \%$. Moreover, the depicted in-plane displacement component based on the present theory has an excellent agreement with the coupled 3D finite element results. The model estimates the in-plane and 


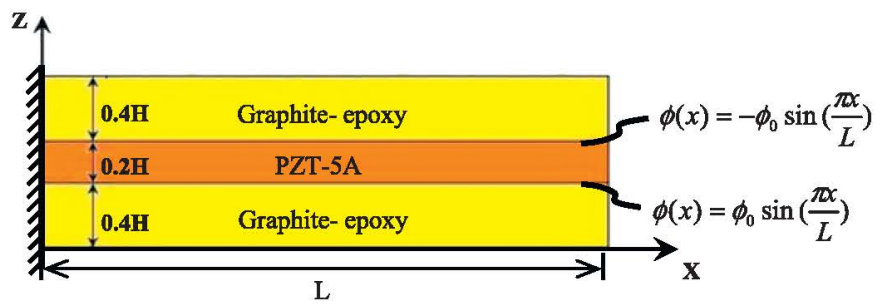

Fig. 16. Characteristics of the geometry of the cantilever sandwich beam Including shear piezoelectric actuator and the actuating potential.
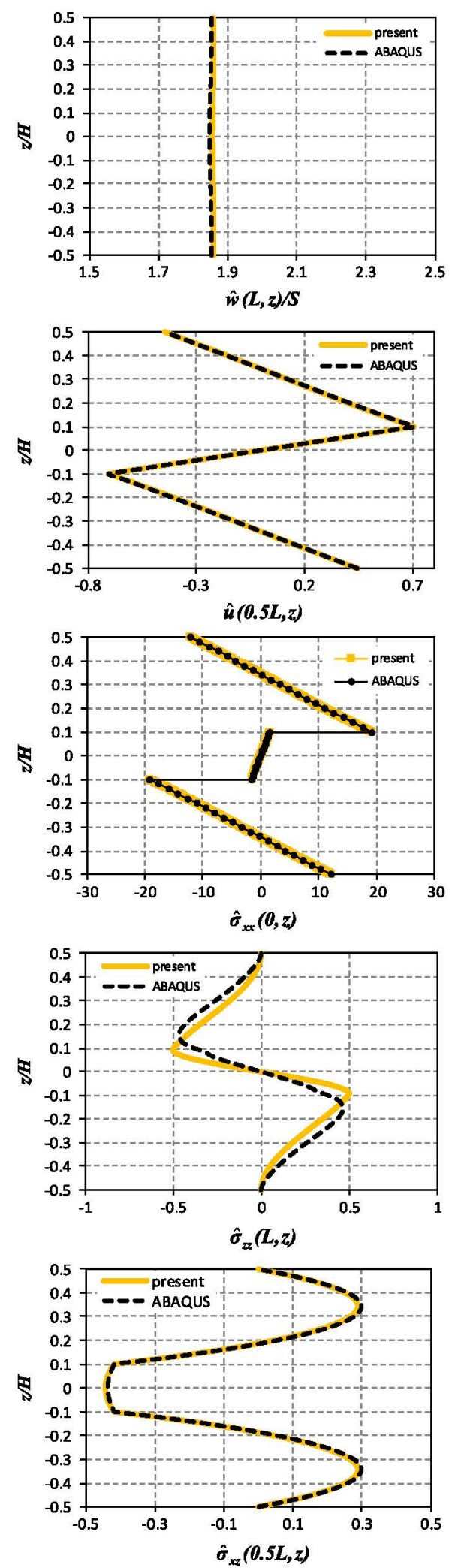

Fig. 17. Variations of the stress and displacement components for the cantilever sandwich beam including shear piezoelectric actuator. 


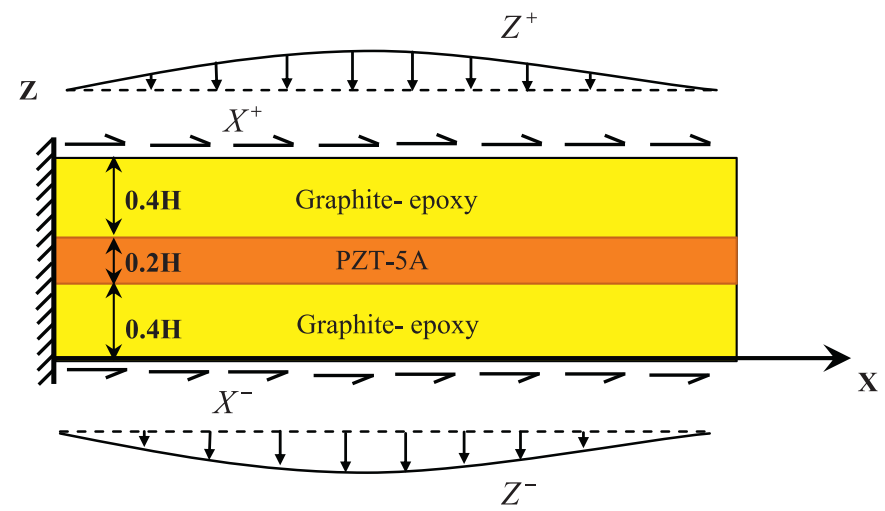

Fig. 18. Description of the geometry, boundary conditions, and loadings of the smart sandwich beam.

transverse shear stresses with a maximum error of $0.4 \%$ and $1.5 \%$, respectively. The effectiveness of the present theory in the prediction of the active behavior of smart sandwich beams is confirmed through these results.

\subsection{Example 3}

In this example, the previous smart sandwich beam with the same geometry, material and boundary conditions is considered. The aforementioned beam is subjected to sinusoidal pressures $Z^{+}=-0.5 \sin (\pi x / L)$ and $Z^{-}=0.5 \sin (\pi x / L)$, and shear tractions $X^{+}=5$ and $X^{-}=-5$ on its top and the bottom surfaces, respectively (Fig. 18).

Through-the-thickness distributions of the stress and displacement components are plotted in Fig. 19. These results are compared with the results of the 3D finite element analysis (ABAQUS) as a benchmark. It may be observed that the displacement components based on the present formulation are in excellent agreement with the finite element results. The error of the present results is $2.17 \%$ for the in-plane displacement and $0.64 \%$ for the transverse displacement. Moreover, the proposed finite element model predicts the in-plane stress with an error less than 8.8\%. As shown in Fig. 19, the prediction of the transverse shear and normal stresses by the present theory agrees well with the results of ABAQUS due to the inclusion of the effects of the non-zero shear and normal tractions. The model predicts the transverse shear and normal stresses of the sensory sandwich beam with an error less than $5 \%$ and $3 \%$, respectively.

\subsection{Example 4}

In order to compare the behavior of beams with shear and extension actuation mechanisms, two sets of beams with small width are considered in this example. The first set concerns beams with shear-mode piezoelectric actuators, while the second set represents beams with extension-mode piezoelectric actuators. The geometric configurations, stacking sequence and poling direction of these two sets of beams are presented in Fig. 20. The piezoelectric layers are made of PZT-5A. The material properties considered for the aluminum are Young's modulus $E=73 \mathrm{GPa}$ and Poission's ratio $v=0.3$. In order to bend the beams, voltages are applied at the top and the bottom surfaces of the piezoelectric layers. For both shear and extension actuation mechanisms, the actuating potential $\phi(x)=1 \mathrm{~V}$ is applied to the top surface of the piezoelectric layer, while the bottom surface is grounded.

The deflection induced by the actuators is calculated for beams with C-F (clamped-free), S-S (simply support) and C-C (clampedclamped) boundary conditions. For these various boundary conditions, the transverse deflection of beams with shear and extension piezoelectric actuators is shown in Fig. 21. Through-the-thickness distribution of the in-plane stress at different sections of beams is also shown in Fig. 22. In case of C-F boundary conditions, it is observed that the tip deflection is slightly smaller for the beams with shear actuation mechanism. However, as can be seen from Fig. 22, the maximum in-plane stress in the shear piezoelectric actuator is about $20 \%$ lower than the extension piezoelectric actuator. The transverse shear and normal stresses for both shear and extension piezoelectric actuators are negligible. It can be observed from Figs. 21 and 22 that unlike the extension actuation mechanism, which cannot produce bending deflection in the $\mathrm{C}-\mathrm{C}$ beam, the shear actuation mechanism can generate deflection in the $\mathrm{C}-\mathrm{C}$ beam at a low stress level. In the case of S-S boundary conditions, the shear actuation mechanism cannot produce bending deflection in the beam.

This example shows that the main advantage provided by the shear actuation mechanism is in its lower stresses in the piezoelectric actuator. Since piezoelectric materials are very brittle, high stresses are detrimental for the structural integrity of the actuator. Moreover, in conditions such as beams with $\mathrm{C}-\mathrm{C}$ boundary conditions, the shear-mode piezoelectric actuators can generate deflection in the host beam.

\section{Conclusions}

A computationally economic and accurate coupled refined global-local finite element model is presented for static response of shear piezoelectric sandwich beams. By using this proposed theory, all kinematic and stress boundary conditions are satisfied at the interfaces of the piezoelectric layers with non-zero longitudinal electric field. Moreover, both electrical transverse normal strains and transverse flexibility are taken into account for the first time in the present theory. Also, the non-zero shear and normal traction boundary conditions on the top and the bottom surfaces of the beam are satisfied for any electrical boundary conditions. The describing expression of the in-plane displacement of the beam contains a high-order polynomial, an exponential expression and a layerwise term containing the electrical unknowns. The transverse displacement is introduced using a combination of continuous piecewise fourth-order polynomial with layerwise components and a first order differentiation of electrical unknowns. A quadratic electric potential is also assumed in the 

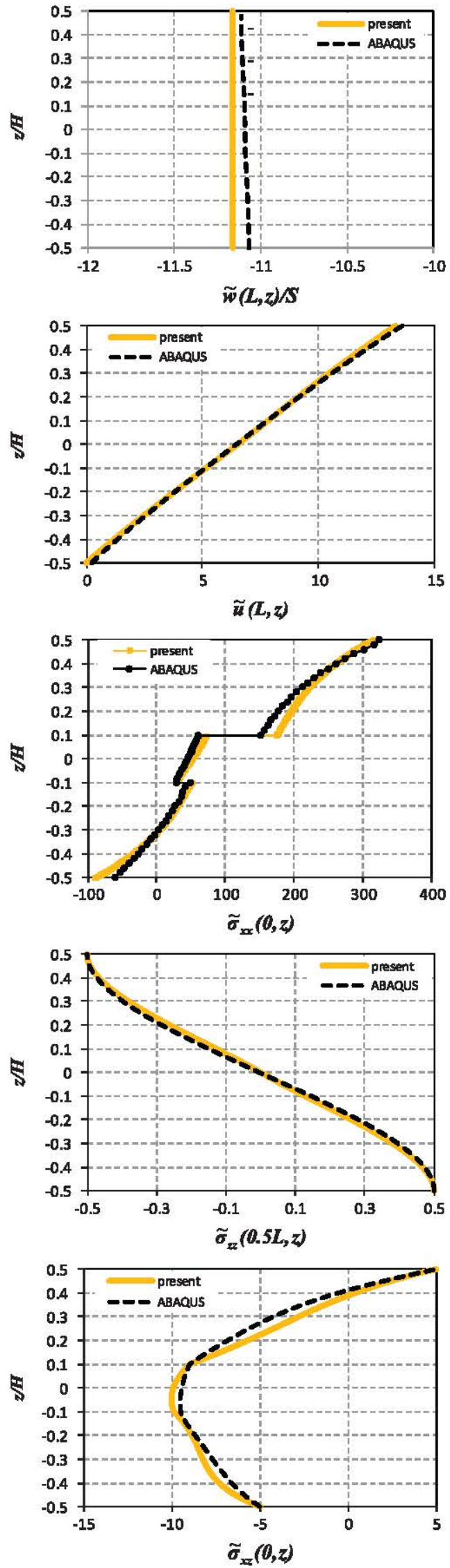

Fig. 19. Variations of the stress and displacement components and the induced transverse electric field for the cantilever sandwich beam 


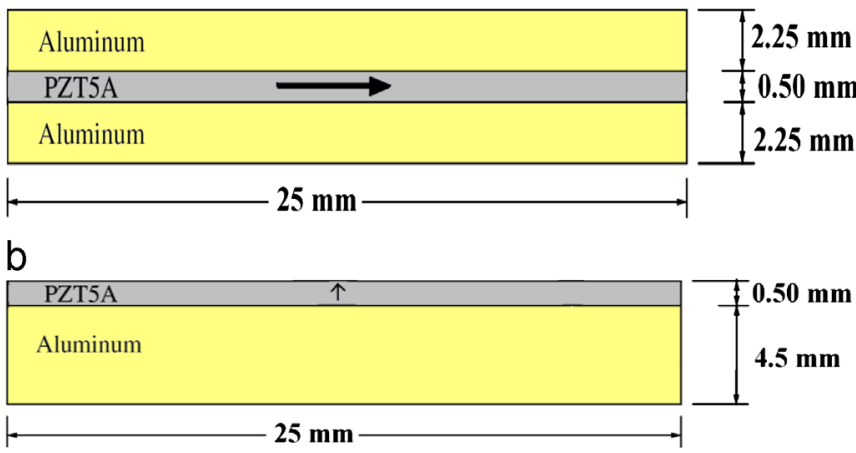

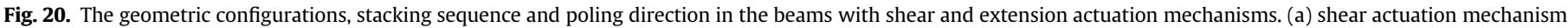
and (b) extension actuation mechanism.
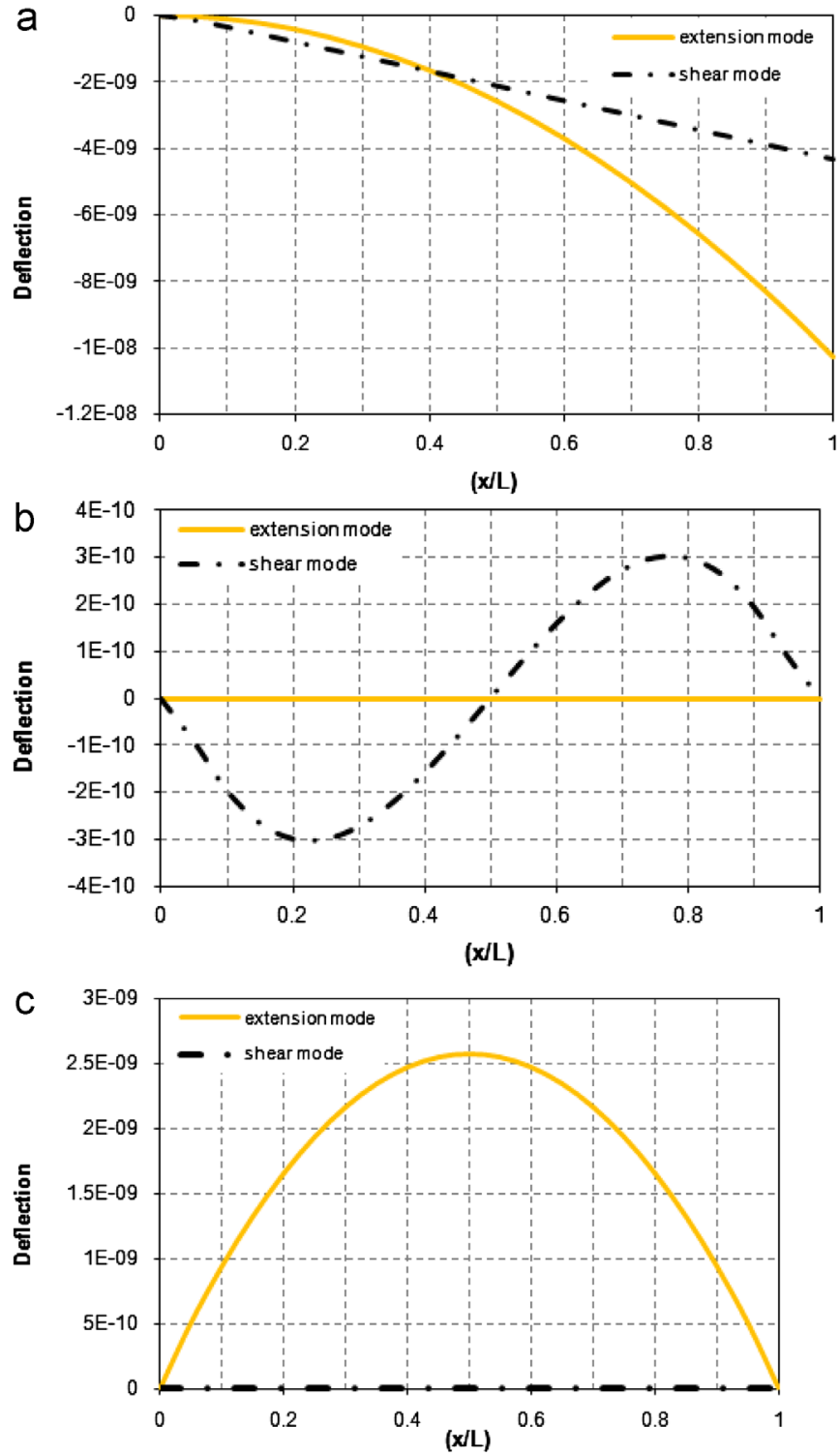

Fig. 21. Transverse deflection of beams with shear and extension mode piezoelectric actuator for: (a) C-F, (b) C-C and (c) S-S boundary conditions.

piezoelectric layers. In the proposed finite element formulation, the mechanical number of the unknown parameters is very small and is independent of the number of the layers. Besides, the shear locking phenomenon does not appear in the proposed smart beam element.

In order to verify the accuracy of the proposed finite element formulation, some comparisons have been made with the results obtained from the coupled 3D finite element (ABAQUS) analysis and 3D theory of piezoelasticity. To this end, various electro-mechanical 

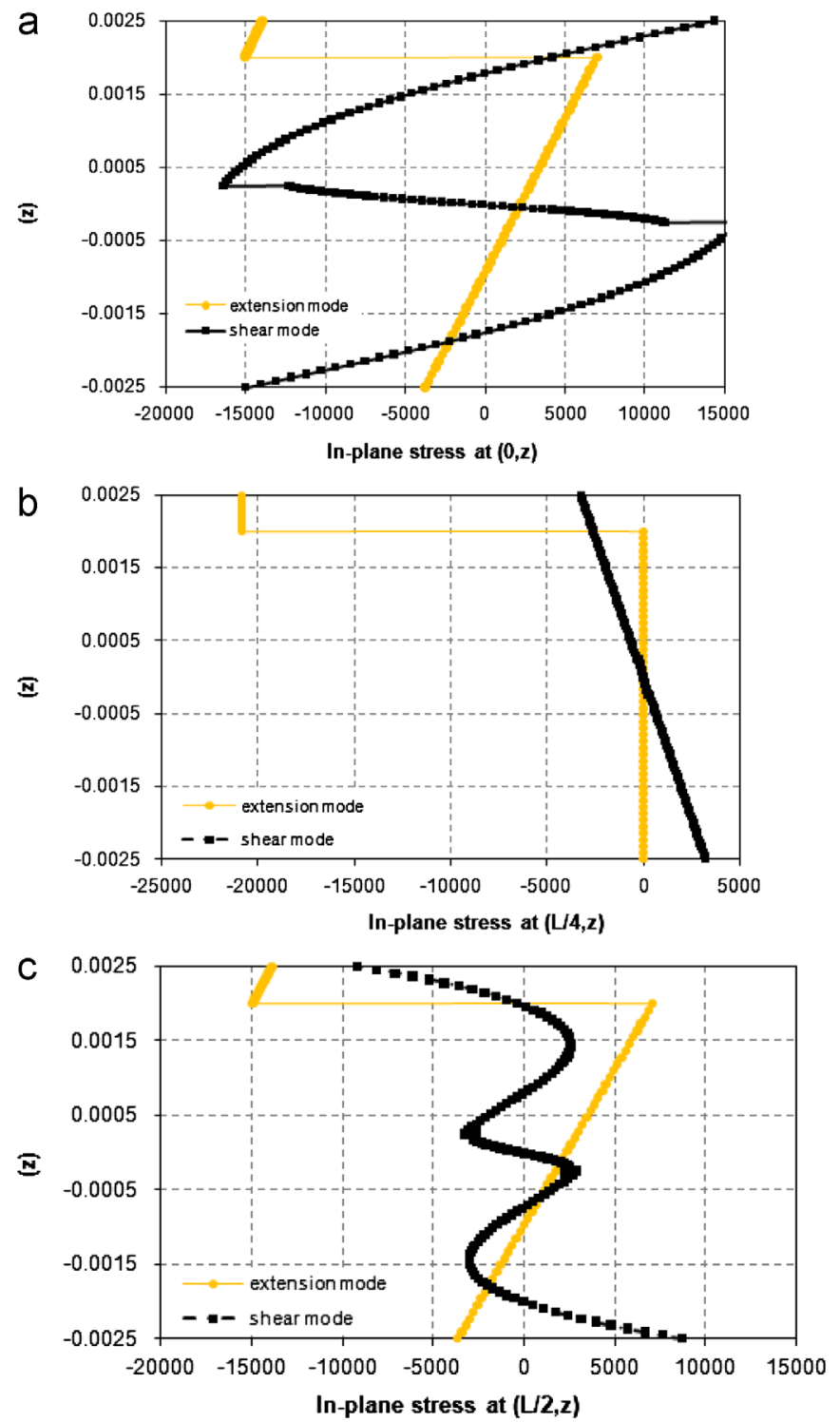

Fig. 22. Through-the-thickness distribution of the in-plane stress at different sections of beams with shear and extension mode piezoelectric actuator for: (a) C-F, (b) C-C and (c) S-S boundary conditions.

bending tests for shear piezoelectric sandwich beams with different geometric parameters and boundary conditions are considered. The comparisons show that the presented coupled finite element formulation, besides its advantages of low computational time due to using small number of the unknown parameters, is sufficiently accurate in the modeling of thin and thick piezoelectric sandwich beams under different mechanical and electrical loading conditions. The presented theory not only shows its superiority for thin beams $(S=40)$ but also presents its higher level of accuracy for thick beams $(S=4)$.

\section{References}

[1] Crawley EF, Luis J. Use of piezoelectric actuators as element of intelligent structures. AIAA Journal 1987;25:1373-85.

[2] Tzou HS, Gadre M. Theoretical analysis of a multi-layered thin shell coupled with piezoelectric shell actuators for distributed vibration controls. Journal of Sound and Vibration 1989;450:132-433.

[3] Wang BT, Rogers CA. Laminate plate theory for spatially distributed induced strain actuators. Journal of Composite Materials 1991;25(4):433-52.

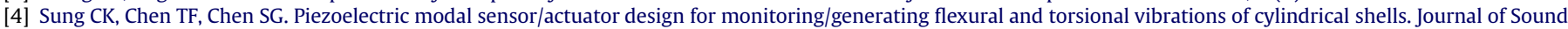
and Vibration 1996;118:48-55.

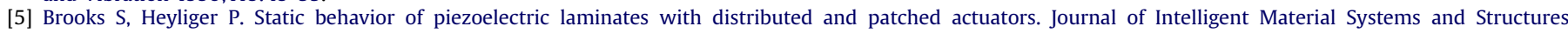
1994;5:635-46.

[6] Ray MC, Rao KM, Samanta B. Exact analysis of coupled electroelastic behavior of a piezoelectric plate under cylindrical bending. Journal of Computers and Structures 1992;45(4):667-77.

[7] Ray MCH, Rao KM, Samanta B. Exact solution for static analysis of an intelligent structure under cylindrical bending. Journal of Computers and Structures 1993;47 (6):1031-42.

[8] Hwang WS, Park HC. Finite element modeling of piezoelectric sensors and actuators. AIAA Journal 1993;31:930-7.

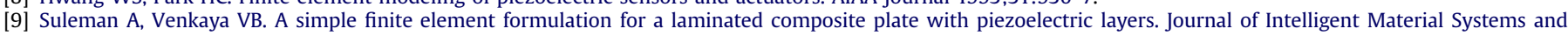
Structures 1995;6:776-82.

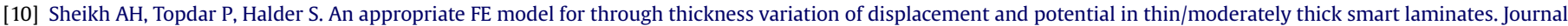
of Computers and Structures 2001;51:401-9. 
[11] Kogl M, Bucalem ML. A family of piezoelectric MITC plate elements. Journal of Computers and Structures 2005;83:1277-97.

[12] Kogl M, Bucalem ML. Analysis of smart laminates using piezoelectric MITC plate and shell elements. Journal of Computers and Structures 2005;83:1153-63.

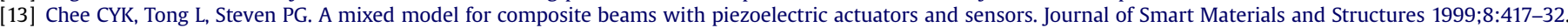

[14] Jiang JP, Li DX. A new finite element model for piezothermoelastic composite beam. Journal of Sound and Vibration 2007;306:849-64.

[15] Shu X. Free-vibration of laminated piezoelectric composite plates based on an accurate theory. Journal of Computers and Structures 2005;67:375-82.

[16] Thornburgh RP, Chattopadhyay A. Simultaneous modeling of mechanical and electrical response of smart composite structures. AIAA Journal 2002;40(8):1603-10.

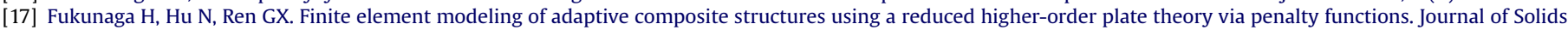
and Structures 2001;38:8735-52.

[18] Mitchell JA, Reddy JN. A refined plate theory for composite laminates with piezoelectric laminate. Journal of Solids and Structures 1995;32(16):2345-67.

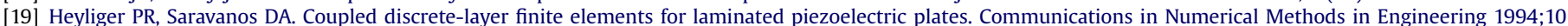
(12):971-81.

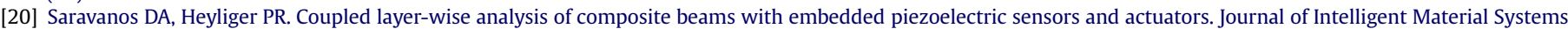
and Structures 1995;6:350-63.

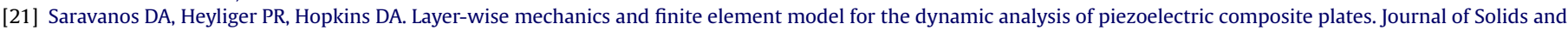
Structures 1997;34(3):359-78.

[22] Kusculuoglu ZK, Fallahi B, Royston TY. Finite element model of a beam with a piezoelectric patch actuator. Journal of Sound and Vibration 2004;276:27-44.

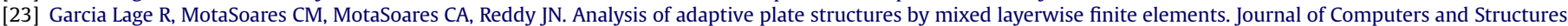
2004;66:269-76.

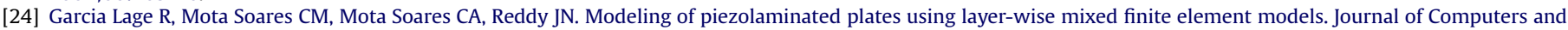
Structures 2004;82:1849-63.

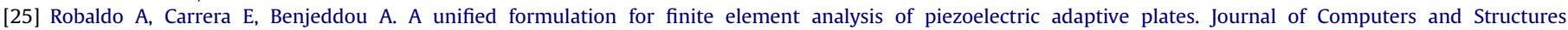
2006;84:1494-505.

[26] Tzou HS, Ye R. Analysis of piezoelastic structures with laminated piezoelectric triangle shell elements. AIAA Journal 1996110-5.

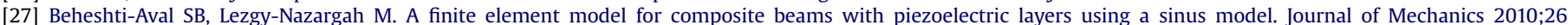
(2):249-58.

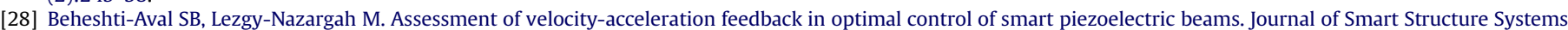
2010;6(8):921-38

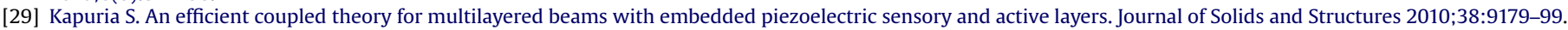

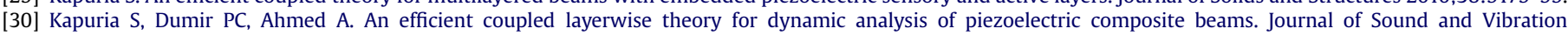
2003:261:927-44.

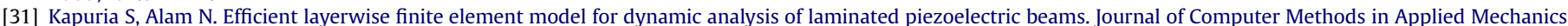
and Engineering 2006;195:2742-60.

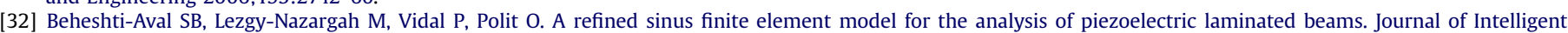
Material Systems and Structures 2011:22:203-19.

[33] Benjeddou A. Advances in piezoelectric finite element modeling of adaptive structural elements: a survey. Journal of Computers and Structures 2000;76:347-63.

[34] Sun CT, Zhang XD. Use of thickness-shear mode in adaptive sandwich structures. Journal of Smart Structure Systems 1995;4:202-6.

[35] Zhang XD, Sun CT. Formulation of an adaptive sandwich beam. Journal of Smart Materials and Structures 1996;5:814-23.

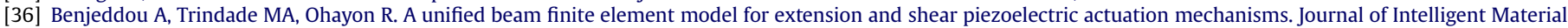
Systems and Structures 1997;8:1012-25.

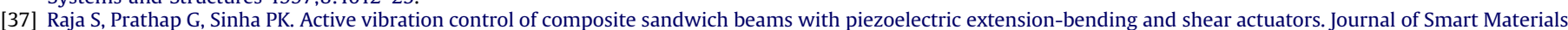
and Structures 2002;11:63-71.

[38] Aldraihem OJ, Khdeir AA. Smart beams with extension and thickness-shear piezoelectric actuators. Journal of Smart Materials and Structures 2000;9:1-9.

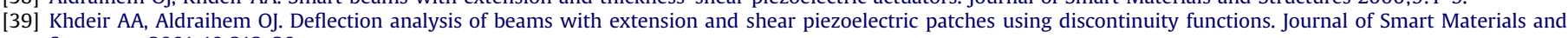
Structures 2001;10:212-20.

[40] Aldraihem OJ, Khdeir AA. Exact deflection solutions of beams with shear piezoelectric actuators. Journal of Solids and Structures 2003;40:1-12.

[41] Aldraihem OJ, Khdeir AA. Precise deflection analysis of beams with piezoelectric patches. Journal of Composite Structures 2003;60:135-43.

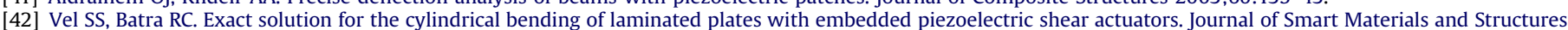
2001;10:240-51.

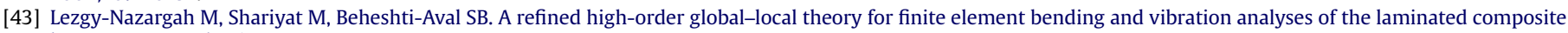
beams. Acta Mechanica 2011;217:3-4.

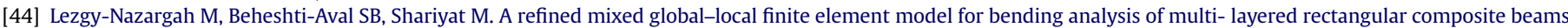
with small widths. Thin Walled Structures 2011;49:351-62. 